\title{
Phenomenology of a little Higgs pseudoaxion
}

\author{
Kingman Cheung, ${ }^{1,2,3, *}$ Shi-Ping He, ${ }^{4, \dagger}$ Ying-nan Mao, ${ }^{1,5, *}$ Po-Yan Tseng, ${ }^{6,8}$ and Chen Zhang ${ }^{1, \|}$ \\ ${ }^{1}$ Physics Division, National Center for Theoretical Sciences, Hsinchu, Taiwan 300 \\ ${ }^{2}$ Department of Physics, National Tsing Hua University, Hsinchu 300, Taiwan \\ ${ }^{3}$ Division of Quantum Phases and Devices, School of Physics, Konkuk University, \\ Seoul 143-701, Republic of Korea \\ ${ }^{4}$ Institute of Theoretical Physics \& State Key Laboratory of Nuclear Physics and Technology, \\ Peking University, Beijing 100871, China \\ ${ }^{5}$ Center for Future High Energy Physics \& Theoretical Physics Division, Institute of High Energy Physics, \\ Chinese Academy of Sciences, Beijing 100049, China \\ ${ }^{6}$ Kavli IPMU (WPI), UTIAS, The University of Tokyo, Kashiwa, Chiba 277-8583, Japan
}

(Received 19 September 2018; published 29 October 2018)

\begin{abstract}
In models where the Higgs is realized as a pseudo-Nambu-Goldstone boson (pNGB) of some global symmetry breaking, there are often remaining pNGBs of some $U(1)$ groups (called "pseudoaxions") which could lead to smoking-gun signatures of such scenarios and provide important clues on the electroweak symmetry breaking mechanism. As a concrete example, we investigate the phenomenology of the pseudoaxion in the anomaly-free simplest little Higgs (SLH) model. After clarifying a subtle issue related to the effect of symmetric vector-scalar-scalar vertices [e.g., $Z_{\mu}\left(H \partial^{\mu} \eta+\eta \partial^{\mu} H\right)$ ], we show that for a natural region in the parameter space, the SLH pseudoaxion is top-philic, decaying almost exclusively to a pair of top quarks. The direct and indirect (i.e., via heavy particle decay) production of such a pseudoaxion at the $14 \mathrm{TeV}$ (HL-)LHC turn out to suffer from either large backgrounds or small rates, making its detection quite challenging. A $p p$ collider with higher energy and luminosity, such as the $27 \mathrm{TeV}$ HE-LHC, or even the $100 \mathrm{TeV}$ FCC-hh or SppC, is therefore motivated to capture the trace of such a pNGB.
\end{abstract}

DOI: 10.1103/PhysRevD.98.075023

\section{INTRODUCTION}

Despite the great success of the Standard Model (SM), marked by the discovery of the $125 \mathrm{GeV}$ Higgs-like boson $[1,2]$ and the ongoing measurements of its properties, how the SM is embedded into a larger theory still remains a mystery. Since the Higgs boson mass parameter is in general not protected under radiative correction, a naive embedding would signal a high sensitivity of infrared (IR) parameters (the electroweak scale and the Higgs boson mass) to ultraviolet (UV) parameters (i.e., physical parameters defined at a high scale). Although this fine-tuned situation is logically possible, or might be explained to some extent by anthropic reasoning [3,4], it is nevertheless

\footnotetext{
*cheung@phys.nthu.edu.tw

†sphe@pku.edu.cn

¥ynmao@cts.nthu.edu.tw

\$poyen.tseng@ipmu.jp

czhang@cts.nthu.edu.tw
}

Published by the American Physical Society under the terms of the Creative Commons Attribution 4.0 International license. Further distribution of this work must maintain attribution to the author(s) and the published article's title, journal citation, and DOI. Funded by SCOAP ${ }^{3}$. natural to conjecture the existence of some systematic mechanism which protects the Higgs boson mass parameter from severe radiative instability. A well-known example of such a systematic mechanism is supersymmetry, which has the merit of being weakly coupled and thus offers better calculability compared to scenarios based on strong dynamics. However, supersymmetry requires the introduction of a large number of new degrees of freedom (d.o.f.), and a large number of new parameters associated with them, making the model quite cumbersome. None of the new d.o.f. have been observed. It is therefore well motivated to consider alternative but simpler mechanisms with weakly coupled dynamics in their range of validity.

One candidate of such an alternative is the little Higgs mechanism [5-8], ${ }^{1}$ in which the Higgs boson is a Goldstone boson of some spontaneous global symmetry breaking. The global symmetry is also explicitly broken in a collective manner ${ }^{2}$ such that the Higgs boson acquires a

\footnotetext{
${ }^{1}$ We refer the reader to Refs. [9,10] for reviews of little Higgs models and Refs. [11-14] for some recent phenomenological analyses of little Higgs models.

${ }^{2}$ More specifically, the global symmetry is completely (explicitly) broken by a collection of spurions but not by any single spurion [8].
} 
mass and at the same time the model is radiatively more stable. A very simple implementation of this collective symmetry breaking (CSB) idea is the simplest little Higgs (SLH) model $[15,16]$, in which the electroweak gauge group is enlarged to $S U(3)_{L} \times U(1)_{X}$, and two scalar triplets are introduced to realize the global symmetrybreaking pattern

$$
\begin{aligned}
& {\left[S U(3)_{1} \times U(1)_{1}\right] \times\left[S U(3)_{2} \times U(1)_{2}\right]} \\
& \rightarrow\left[S U(2)_{1} \times U(1)_{1}\right] \times\left[S U(2)_{2} \times U(1)_{2}\right] .
\end{aligned}
$$

The global symmetry is also explicitly broken by gauge and Yukawa interactions, but in a collective manner to improve the radiative stability of the scalar sector. The particle content is quite economical. In particular, in the low-energy scalar sector there exists only two physical d.o.f., one of which (denoted $H$ ) could be identified with the $125 \mathrm{GeV}$ Higgs-like particle, while the other is a $C P$-odd scalar $\eta$ which is referred to as a pseudoaxion in the literature $[17,18]$.

In the SLH, the pseudoaxion $\eta$ is closely related to the electroweak symmetry breaking (EWSB) and therefore studying its phenomenology is well motivated. According to the hidden mass relation derived in Ref. [19], the $\eta$ mass $m_{\eta}$ is anticorrelated with the top partner mass $m_{T}$, which is in turn related to the degree of fine-tuning in the model. The hidden mass relation is derived within an approach consistent with the continuum effective field theory (CEFT) and does not rely on the assumption on the contribution from the physics at the cutoff. Although the phenomenology of the $\eta$ particle has been studied in quite a few papers (e.g., Refs. $[14,17,18,20,21])$, their treatment was not based on the hidden mass relation, and also most of the papers were written before the $125 \mathrm{GeV}$ boson was discovered. It is thus timely to revisit the status of $\eta$ phenomenology in light of the discovery of the $125 \mathrm{GeV}$ boson, taking into account the properly derived hidden mass relation and focusing on the parameter space favored by naturalness considerations.

There is another important reason that warrants a reanalysis of the $\eta$ phenomenology. The SLH is usually written as a gauged nonlinear sigma model, in which the EWSB can be parametrized through vacuum misalignment. However, the vacuum misalignment also leads to the fact that, in the usual parametrization of the two scalar triplets, there exist scalar kinetic terms that are not canonically normalized, and vector-scalar two-point transitions that are "unexpected" [22]. A further field rotation, including an appropriate gauge-fixing procedure, is thus required to properly diagonalize the vector-scalar sector of the SLH model. This subtlety was overlooked in all related papers before Ref. [22], and if one carries out a proper diagonalization of the bosonic sector of the SLH, some of the $\eta$-related couplings will turn out to be different from what has been obtained in previous literature. This is the case for both the $\mathrm{ZH} \eta$ coupling and the coupling of $\eta$ to a pair of $\mathrm{SM}$ fermions. The occurrence of the mass eigenstate antisymmetric $Z H \eta$ vertex [i.e., $Z_{\mu}\left(H \partial^{\mu} \eta-\eta \partial^{\mu} H\right)$ ] is postponed to $\mathcal{O}\left(\xi^{3}\right)$ [where $\xi \equiv \frac{v}{f}, v \approx 246 \mathrm{GeV}$ and $f$ is the global symmetry-breaking scale of Eq. (1)], and the couplings of $\eta$ to a pair of SM charged leptons, and to $b \bar{b}$, $c \bar{c}, u \bar{u}$ are found to vanish to all orders in $\xi$. This leads to significant changes in the $\eta$ phenomenology, which will be studied in detail in this work.

When one tries to derive the $\eta$-related Lagrangian in the SLH, symmetric vector-scalar-scalar (VSS) vertices, e.g., $Z_{\mu}\left(H \partial^{\mu} \eta+\eta \partial^{\mu} H\right)$ naturally appear, which is a feature that is often present in models based on a nonlinearly realized scalar sector. The effects of such symmetric VSS vertices contain some subtleties which, to our knowledge, have not been discussed before in the literature. Therefore, we devote one section to the analysis of symmetric VSS vertices, which could also be helpful to clarify similar situations in other nonlinearly realized models.

In this work we do not aim to give a complete characterization of the $\eta$ phenomenology, which could be very complicated in certain corners of parameter space. Instead, we focus our attention on the parameter space favored by naturalness considerations. More specifically, we will consider an $\eta$ mass in the region $2 m_{t} \lesssim m_{\eta} \lesssim 1 \mathrm{TeV}$, which is favored by naturalness. We then calculate the $\eta$ decay and production at future high-energy hadron colliders in various channels. It turns out that at the $14 \mathrm{TeV}$ (HL)-LHC the detection of $\eta$ is quite challenging due to various suppression mechanisms. A $p p$ collider with higher energy and luminosity, such as the $27 \mathrm{TeV}$ HE-LHC, or even the $100 \mathrm{TeV}$ FCC-hh or SppC, is therefore motivated to capture the trace of such a pNGB.

The paper is organized as follows. In Sec. II we review the basic ingredients of the SLH, including the crucial hidden mass relation obtained from a CEFT analysis, and present the mass eigenstate Lagrangian relevant for phenomenological studies. In Sec. III we clarify the effect of symmetric VSS vertices. Then, in Sec. IV we derive important constraints from electroweak precision observables relevant for the pseudoaxion phenomenology. Section $\mathrm{V}$ is dedicated to the study of $\eta$ decay and production at hadron colliders. In Sec. VI we present the discussion and conclusions.

\section{THE SIMPLEST LITTLE HIGGS}

\section{A. Overview of the simplest little Higgs}

In the $\mathrm{SLH}$, the electroweak gauge group is enlarged to $S U(3)_{L} \times U(1)_{X}$. Two scalar triplets $\Phi_{1}, \Phi_{2}$ are introduced to realize the spontaneous global symmetry breaking pattern in Eq. (1). They are parametrized as

$$
\Phi_{1}=\exp \left(\frac{i \Theta^{\prime}}{f}\right) \exp \left(\frac{i t_{\beta} \Theta}{f}\right)\left(\begin{array}{c}
0 \\
0 \\
f c_{\beta}
\end{array}\right),
$$




$$
\Phi_{2}=\exp \left(\frac{i \Theta^{\prime}}{f}\right) \exp \left(-\frac{i \Theta}{f t_{\beta}}\right)\left(\begin{array}{c}
0 \\
0 \\
f s_{\beta}
\end{array}\right)
$$

Here we have introduced the shorthand notation $s_{\beta} \equiv \sin \beta$, $c_{\beta} \equiv \cos \beta, t_{\beta} \equiv \tan \beta . f$ is the Goldstone decay constant. $\Theta$ and $\Theta^{\prime}$ are $3 \times 3$ matrix fields, parametrized as

$$
\Theta=\frac{\eta}{\sqrt{2}}+\left(\begin{array}{cc}
\mathbf{0}_{2 \times 2} & h \\
h^{\dagger} & 0
\end{array}\right), \quad \Theta^{\prime}=\frac{\zeta}{\sqrt{2}}+\left(\begin{array}{cc}
\mathbf{0}_{2 \times 2} & k \\
k^{\dagger} & 0
\end{array}\right) .
$$

$\eta$ is the pseudoaxion, and $h$ and $k$ are parametrized as [ $v$ denotes the vacuum expectation value (VEV) of the Higgs doublet]

$$
\begin{gathered}
h=\left(\begin{array}{c}
h^{0} \\
h^{-}
\end{array}\right), \quad h^{0}=\frac{1}{\sqrt{2}}(v+H-i \chi), \\
k=\left(\begin{array}{c}
k^{0} \\
k^{-}
\end{array}\right), \quad k^{0}=\frac{1}{\sqrt{2}}(\sigma-i \omega) .
\end{gathered}
$$

For future convenience, we introduce the notation

$$
\hat{h} \equiv\left(h^{\dagger} h\right)^{1 / 2}
$$

We note that the spontaneous global symmetry breaking (1) should deliver ten Goldstone bosons, which are parametrized here in terms of $\Theta$ and $\Theta^{\prime}$. The electroweak gauge group $S U(3)_{L} \times U(1)_{X}$ will eventually break to $U(1)_{E M}$, and therefore eight Goldstone bosons will be eaten to make the associated gauge bosons massive. Only two Goldstone bosons remain physical, denoted here as $h$ and $\eta$. The parametrization of these Goldstone fields actually has some freedom; we refer the reader to Ref. [19] for an explanation.

In the SLH, under the full gauge group $S U(3)_{C} \times$ $S U(3)_{L} \times U(1)_{X}, \quad \Phi_{1}$ and $\Phi_{2}$ have quantum number $(\mathbf{1}, \mathbf{3})_{-\frac{1}{3}}$. The gauge kinetic term of $\Phi_{1}$ and $\Phi_{2}$ can thus be written as $^{3}$

$$
\mathcal{L}_{g k}=\left(D_{\mu} \Phi_{1}\right)^{\dagger}\left(D^{\mu} \Phi_{1}\right)+\left(D_{\mu} \Phi_{2}\right)^{\dagger}\left(D^{\mu} \Phi_{2}\right),
$$

in which the covariant derivative can be expressed as ${ }^{4}$

$$
D_{\mu}=\partial_{\mu}-i g A_{\mu}^{a} T^{a}+i g_{x} Q_{x} B_{\mu}^{x}, \quad g_{x}=\frac{g t_{W}}{\sqrt{1-t_{W}^{2} / 3}}
$$

\footnotetext{
${ }^{3}$ We note that Eq. (8) automatically satisfies the requirement of CSB.

${ }^{4}$ In this paper our convention agrees with Ref. [23] but differs from Ref. [24]. The conversion between the two conventions is discussed in Appendix A.
}

In the above equation, $A_{\mu}^{a}$ and $B_{\mu}^{x}$ denote $S U(3)_{L}$ and $U(1)_{X}$ gauge fields, respectively. $g$ and $g_{x}$ denote the coupling constants of $S U(3)_{L}$ and $U(1)_{X}$ gauge groups, respectively. It is convenient to trade $g_{x}$ for $t_{W} \equiv \tan \theta_{W} . T^{a}=\frac{\lambda^{a}}{2}$ where $\lambda^{a}, a=1, \ldots, 8$ denote the Gell-Mann matrices. For $\Phi_{1}$, $\Phi_{2}, Q_{x}=-\frac{1}{3}$. Following Ref. [23], we parametrize the $S U(3)_{L}$ gauge bosons as

$$
\begin{aligned}
A_{\mu}^{a} T^{a}= & \frac{A_{\mu}^{3}}{2}\left(\begin{array}{ccc}
1 & 0 & 0 \\
0 & -1 & 0 \\
0 & 0 & 0
\end{array}\right)+\frac{A_{\mu}^{8}}{2 \sqrt{3}}\left(\begin{array}{ccc}
1 & 0 & 0 \\
0 & 1 & 0 \\
0 & 0 & -2
\end{array}\right) \\
& +\frac{1}{\sqrt{2}}\left(\begin{array}{ccc}
0 & W_{\mu}^{+} & Y_{\mu}^{0} \\
W_{\mu}^{-} & 0 & X_{\mu}^{-} \\
Y_{\mu}^{0 \dagger} & X_{\mu}^{+} & 0
\end{array}\right),
\end{aligned}
$$

with the first-order neutral gauge boson mixing relation $\left(c_{W} \equiv \cos \theta_{W}, s_{W} \equiv \sin \theta_{W}\right)$

$$
\left(\begin{array}{l}
A^{3} \\
A^{8} \\
B^{x}
\end{array}\right)=\left(\begin{array}{ccc}
0 & c_{W} & -s_{W} \\
\sqrt{1-\frac{t_{W}^{2}}{3}} & \frac{s_{W} t_{W}}{\sqrt{3}} & \frac{s_{W}}{\sqrt{3}} \\
-\frac{t_{W}}{\sqrt{3}} & s_{W} \sqrt{1-\frac{t_{W}^{2}}{3}} & c_{W} \sqrt{1-\frac{t_{W}^{2}}{3}}
\end{array}\right)\left(\begin{array}{c}
Z^{\prime} \\
Z \\
A
\end{array}\right) .
$$

Since the electroweak gauge group is enlarged to $S U(3)_{L} \times U(1)_{X}$, it is also necessary to enlarge the fermion sector in order that fermions transform properly under the enlarged group. We adopt the elegant anomaly-free embedding proposed in Refs. $[16,25,26]$. In the lepton Yukawa sector, the SM left-handed lepton doublets are enlarged to $S U(3)_{L}$ triplets $L_{m}=\left(\nu_{L}, \ell_{L}, i N_{L}\right)_{m}^{T}$ with $Q_{x}=-\frac{1}{3}$ ( $m=1,2,3$ is the family index). There are also righthanded singlet lepton fields $\ell_{R m}$ with $Q_{x}=-1$ and $N_{R m}$ with $Q_{x}=0$. The lepton Yukawa Lagrangian can be written as [23]

$\mathcal{L}_{L Y}=i \lambda_{N}^{m} \bar{N}_{R m} \Phi_{2}^{\dagger} L_{m}+\frac{i \lambda_{\ell}^{m n}}{\Lambda} \bar{\ell}_{R m} \epsilon_{i j k} \Phi_{1}^{i} \Phi_{2}^{j} L_{n}^{k}+$ H.c.

In the quark sector, we have the following field content:

$$
\begin{gathered}
Q_{1}=\left(d_{L},-u_{L}, i D_{L}\right)^{T}, \quad d_{R}, \quad u_{R}, \quad D_{R}, \\
Q_{2}=\left(s_{L},-c_{L}, i S_{L}\right)^{T}, \quad s_{R}, \quad c_{R}, \quad S_{R}, \\
Q_{3}=\left(t_{L}, b_{L}, i T_{L}\right)^{T}, \quad t_{R}, \quad b_{R}, \quad T_{R} .
\end{gathered}
$$

Here $Q_{1}, Q_{2}$ transform under the $\overline{\mathbf{3}}$ representation of $S U(3)_{L}$ with $Q_{x}=0 . Q_{3}$ transforms under the 3 representation of $S U(3)_{L}$ with $Q_{x}=\frac{1}{3}$. The right-handed quark fields are all $S U(3)_{L}$ singlets with various $U(1)_{X}$ charges. 
More specifically, $u_{R}, c_{R}, t_{R}, T_{R}$ carry $Q_{x}=\frac{2}{3}$, while $d_{R}, s_{R}$, $b_{R}, D_{R}, S_{R}$ carry $Q_{x}=-\frac{1}{3}$. The quark Yukawa Lagrangian can be written as [23]

$$
\begin{aligned}
\mathcal{L}_{Q Y}= & i \lambda_{1}^{t} \bar{u}_{R 3}^{1} \Phi_{1}^{\dagger} Q_{3}+i \lambda_{2}^{t} \bar{u}_{R 3}^{2} \Phi_{2}^{\dagger} Q_{3} \\
& +i \frac{\lambda_{b}^{m}}{\Lambda} \bar{d}_{R m} \epsilon_{i j k} \Phi_{1}^{i} \Phi_{2}^{j} Q_{3}^{k}+i \lambda_{1}^{d n} \bar{d}_{R n}^{1} Q_{n}^{T} \Phi_{1} \\
& +i \lambda_{2}^{d n} \bar{d}_{R n}^{2} Q_{n}^{T} \Phi_{2}+i \frac{\lambda_{u}^{m n}}{\Lambda} \bar{u}_{R m} \epsilon_{i j k} \Phi_{1}^{* i} \Phi_{2}^{* j} Q_{n}^{k}+\text { H.c. }
\end{aligned}
$$

In the above equation, $n=1,2$ is the family index for the first two generations of quark triplets. $d_{R m}$ runs over $\left(d_{R}, s_{R}, b_{R}, D_{R}, S_{R}\right)$ and $u_{R m}$ runs over $\left(u_{R}, c_{R}, t_{R}, T_{R}\right)$. $u_{R 3}^{1}, u_{R 3}^{2}$ are linear combinations of $t_{R}$ and $T_{R} \cdot d_{R n}^{1}, d_{R n}^{2}$ are linear combinations of $d_{R}$ and $D_{R}$ for $n=1$ and of $s_{R}$ and $S_{R}$ for $n=2$. It is worth noting that in the dimension-four part of Eqs. (12) and (16) CSB is formally preserved. In contrast, in Eqs. (12) and (16), the dimension-five part formally violates CSB. Nevertheless, the amount of violation is proportional to light fermion Yukawas and is thus negligible.

We now turn to the scalar potential. Using a CEFT approach and combining tree-level ${ }^{5}$ and one-loop contributions, the scalar effective potential in the SLH is calculated to be [19]

$V=-\mu^{2}\left(\Phi_{1}^{\dagger} \Phi_{2}+\Phi_{2}^{\dagger} \Phi_{1}\right)+\lambda\left|\Phi_{1}^{\dagger} \Phi_{2}\right|^{2}+\Delta(\hat{h}) \hat{h}^{4}$.

$\mu^{2}$ and $\lambda$ could be regarded as parameters to be determined from experiments, while $\Delta(\hat{h})$ is automatically finite, and could be expressed in terms of the Lagrangian parameters in the model,

$$
\begin{aligned}
\Delta(\hat{h})= & \frac{3}{16 \pi^{2}}\left\{\lambda_{t}^{4}\left[\ln \frac{M_{T}^{2}}{m_{t}^{2}(\hat{h})}-\frac{1}{2}\right]\right. \\
& -\frac{1}{8} g^{4}\left[\ln \frac{M_{X}^{2}}{m_{W}^{2}(\hat{h})}-\frac{1}{2}\right] \\
& \left.-\frac{1}{16} g^{4}\left(1+t_{W}^{2}\right)^{2}\left[\ln \frac{M_{Z^{\prime}}^{2}}{m_{Z}^{2}(\hat{h})}-\frac{1}{2}\right]\right\} .
\end{aligned}
$$

$\lambda_{t}$ is defined as

$$
\lambda_{t} \equiv \frac{\lambda_{1}^{t} \lambda_{2}^{t}}{\sqrt{\lambda_{1}^{t 2} c_{\beta}^{2}+\lambda_{2}^{t 2} s_{\beta}^{2}}},
$$

\footnotetext{
${ }^{5}$ At tree level we do not include a $\left(\Phi_{1}^{\dagger} \Phi_{2}\right)^{2}+$ H.c. term because it formally violates CSB. We note that introducing such a term may lead to spontaneous $C P$ violation [27]. Furthermore, if both the $\left(\Phi_{1}^{\dagger} \Phi_{2}\right)^{2}+$ H.c. term and Majorana mass terms for $N_{R}$ 's are introduced, the SLH light neutrino masses can be radiatively generated [28].
}

where $\lambda_{1}^{t}, \lambda_{2}^{t}$ are the two Yukawa couplings in the top sector, introduced in Eq. (16). $M_{T}^{2}, M_{X}^{2}, M_{Z^{\prime}}^{2}$ are defined as

$$
\begin{gathered}
M_{T}^{2} \equiv\left(\lambda_{1}^{t 2} c_{\beta}^{2}+\lambda_{2}^{t 2} s_{\beta}^{2}\right) f^{2}, \\
M_{X}^{2} \equiv \frac{1}{2} g^{2} f^{2}, \\
M_{Z^{\prime}}^{2} \equiv \frac{2}{3-t_{W}^{2}} g^{2} f^{2} .
\end{gathered}
$$

They are related to the physical mass squared of the relevant particles as follows:

$$
\begin{gathered}
M_{T}^{2}=m_{T}^{2}+m_{t}^{2}, \\
M_{X}^{2}=m_{X}^{2}+m_{W}^{2}, \\
M_{Z^{\prime}}^{2}=m_{Z^{\prime}}^{2}+m_{Z}^{2},
\end{gathered}
$$

in which $m_{T}, m_{t}$ denote the physical mass of the heavy top $T$ and the top quark $t, m_{X}, m_{W}$ denote the physical mass of the $X$ boson and $W$ boson, and $m_{Z^{\prime}}, m_{Z}$ denote the physical mass of the $Z^{\prime}$ boson and $Z$ boson, respectively. $m_{t}^{2}(\hat{h})$, $m_{W}^{2}(\hat{h}), m_{Z}^{2}(\hat{h})$ are field-dependent masses squared, for which we use the following leading-order (LO) expressions:

$$
\begin{gathered}
m_{t}^{2}(\hat{h})=\lambda_{t}^{2} \hat{h}^{2}, \\
m_{W}^{2}(\hat{h})=\frac{1}{2} g^{2} \hat{h}^{2}, \\
m_{Z}^{2}(\hat{h})=\frac{1}{2} g^{2}\left(1+t_{W}^{2}\right) \hat{h}^{2} .
\end{gathered}
$$

With the above expressions for the scalar effective potential we are able to compute the electroweak VEV, Higgs mass, pseudoaxion mass, etc., as functions of $\mu^{2}, \lambda$, and other Lagrangian parameters in the model.

Finally, we note that there of course exists a gaugeinvariant kinetic Lagrangian for the $S U(3)_{L} \times U(1)_{X}$ gauge fields and the fermion fields in the model, according to their representations.

\section{B. Hidden mass relation, unitarity, and naturalness}

Before starting the phenomenological analysis in the $\mathrm{SLH}$, it is important to notice that there exist certain constraints that we have to take into account [19].

First, there exists a hidden mass relation which follows from an analysis of the scalar effective potential (17). This is because if we consider $g, t_{W}, \lambda_{t}$ as fixed, then the scalar effective potential (17) is fully determined by five parameters, say, $\mu^{2}, \lambda, f, t_{\beta}, m_{T}$. Requiring the electroweak VEV to be $246 \mathrm{GeV}$ and the $C P$-even Higgs mass to be $125 \mathrm{GeV}$ should eliminate two parameters, leaving only three 
parameters as independent. For instance, we may choose $f$, $t_{\beta}, m_{T}$ as the three independent parameters; then, any other observable could be expressed in terms of these three parameters. In particular, the pseudoaxion mass $m_{\eta}$ is determined from the following hidden mass relation derived in Ref. [19]:

$m_{\eta}^{2}=\left[m_{h}^{2}-v^{2} \Delta_{A}\left(3-2 \theta t_{2 \theta}^{-1}\right)+v^{2} A\left(5-2 \theta t_{2 \theta}^{-1}\right)\right] s_{\theta}^{-2}$.

Here $t_{2 \theta}^{-1} \equiv \frac{1}{\tan (2 \theta)}, s_{\theta}^{-2} \equiv \frac{1}{\sin ^{2} \theta}$, and $\theta, A, \Delta_{A}$ are defined by

$$
\begin{gathered}
\theta \equiv \frac{v}{\sqrt{2} f s_{\beta} c_{\beta}}, \\
A \equiv \frac{3}{16 \pi^{2}}\left[\lambda_{t}^{4}-\frac{g^{4}}{8}-\frac{g^{4}}{16}\left(1+t_{W}^{2}\right)^{2}\right], \\
\Delta_{A} \equiv \frac{3}{16 \pi^{2}}\left[\lambda_{t}^{4} \ln \frac{M_{T}^{2}}{m_{t}^{2}}-\frac{g^{4}}{8} \ln \frac{M_{X}^{2}}{m_{W}^{2}}\right. \\
\left.-\frac{g^{4}}{16}\left(1+t_{W}^{2}\right)^{2} \ln \frac{M_{Z^{\prime}}^{2}}{m_{Z}^{2}}\right] .
\end{gathered}
$$

The basic feature of this mass relation is that the pseudoaxion mass is anticorrelated with the top partner mass.

Second, the SLH is meant to be only an effective field theory valid up to some energy scale, which could be revealed by an analysis of partial-wave unitarity. This was done in Ref. [19] and the unitarity cutoff was determined to be

$$
\Lambda_{U}=\sqrt{8 \pi} \times \min \left\{f c_{\beta}, f s_{\beta}\right\} .
$$

Apart from the lepton Yukawa part, the SLH Lagrangian is manifestly symmetric with respect to the exchange $\Phi_{1} \leftrightarrow \Phi_{2}$ (with the corresponding exchange of all related coefficients), and therefore without loss of generality we may restrict to $t_{\beta} \geq 1$. The resulting formulas have the $t_{\beta} \leftrightarrow \frac{1}{t_{\beta}}$ invariance. Nevertheless, the lepton Yukawa Lagrangian (12) does not share this exchange symmetry, and the $t_{\beta} \leftrightarrow \frac{1}{t_{\beta}}$ invariance could be lost. However, if we do not deal directly with lepton-related vertices, the $t_{\beta} \leftrightarrow \frac{1}{t_{\beta}}$ invariance violation could only come from input parameter corrections, which are all suppressed by $\frac{v^{2}}{f^{2}}[19]$, which is a very small quantity if we consider current bounds on $f$. Therefore in the following, unless otherwise specified, we will assume $t_{\beta} \geq 1$. (Moreover, in Sec. IV we will show that the $t_{\beta}<1$ case is disfavored by electroweak precision measurements for natural regions of parameter space.) Then we can express the unitarity cutoff as

$$
\Lambda_{U}=\sqrt{8 \pi} f c_{\beta}
$$

and we require all particle masses be less than $\Lambda_{U}$. We note that since $\Lambda_{U}$ is determined by the smaller of the triplet VEVs, while $m_{Z^{\prime}}$ is determined by the quadrature of the triplet VEVs, requiring $m_{Z^{\prime}} \leq \Lambda_{U}$ leads to an upper bound on $t_{\beta}$ (besides our assumption $t_{\beta} \geq 1$ )

$$
1 \leq t_{\beta} \leq \sqrt{\frac{4 \pi\left(3-t_{W}^{2}\right)}{g^{2}}-1} \approx 8.9 .
$$

Third, the parameter $M_{T}$ has a lower bound derived simply from the structure of the Yukawa Lagrangian [24]

$$
M_{T} \geq \sqrt{2} \frac{m_{t}}{v} f s_{2 \beta} \approx f s_{2 \beta},
$$

where $s_{2 \beta} \equiv \sin (2 \beta) . M_{T}$ is also bounded from above by either $\Lambda_{U}$ or the requirement that $m_{\eta}^{2}$ obtained from Eq. (29) should be positive.

Finally, from the LHC search for the $Z^{\prime}$ boson in the dilepton channel $[29,30]$, we estimate the lower bound on $f$ as [27]

$$
f \gtrsim 7.5 \mathrm{TeV},
$$

We note that when combined with Eqs. (36) and (35) this also leads to a lower bound on the top partner mass of around $1.7 \mathrm{TeV}$, which is much more stringent than constraints from top partner searches at the LHC.

It is remarkable that the naturalness issue can also be analyzed in a CEFT approach, which was done in Ref. [19]. We define the total degree of fine-tuning at a certain parameter point as

$$
\Delta_{\mathrm{TOT}}=\max \left\{\Delta_{\mathrm{TOT}}^{\mu^{2}}, \Delta_{\mathrm{TOT}}^{\lambda}\right\},
$$

where $\Delta_{\mathrm{TOT}}^{\mu^{2}}, \Delta_{\mathrm{TOT}}^{\lambda}$ are defined by

$$
\Delta_{\mathrm{TOT}}^{\lambda} \equiv\left|\frac{\lambda_{U}}{m_{h}^{2}} \frac{\partial m_{h}^{2}}{\partial \lambda_{U}}\right|, \quad \Delta_{\text {TOT }}^{\mu^{2}} \equiv\left|\frac{\mu_{U}^{2}}{m_{h}^{2}} \frac{\partial m_{h}^{2}}{\partial \mu_{U}^{2}}\right| .
$$

Here $\lambda_{U}, \mu_{U}^{2}$ denote the $\lambda, \mu^{2}$ parameters defined at the unitarity cutoff. The above definitions obviously reflect how the IR parameters (e.g., $m_{h}^{2}$ ) are sensitive to UV parameters (e.g., $\lambda_{U}, \mu_{U}^{2}$ ), and thus may serve as a measure of the degree of fine-tuning in the allowed parameter space. We may follow Ref. [19] to compute the degree of finetuning, and find several general features. One feature which is easy to understand is, generally speaking, with smaller $f$ and $m_{T}$ we could get a smaller degree of fine-tuning.

In Fig. 1 we present the density plot of $\log \Delta_{\text {TOT }}$ in the $m_{\eta}-m_{T}$ plane for $f=8 \mathrm{TeV}$. Only the colored region is allowed by various constraints. From the figure it is clear that the parameter region favored by naturalness considerations is featured by a small $m_{T}$, with $m_{\eta}$ around $500 \mathrm{GeV}$. A light $\eta$, with a mass less than $2 m_{t}$, is unfortunately disfavored. 


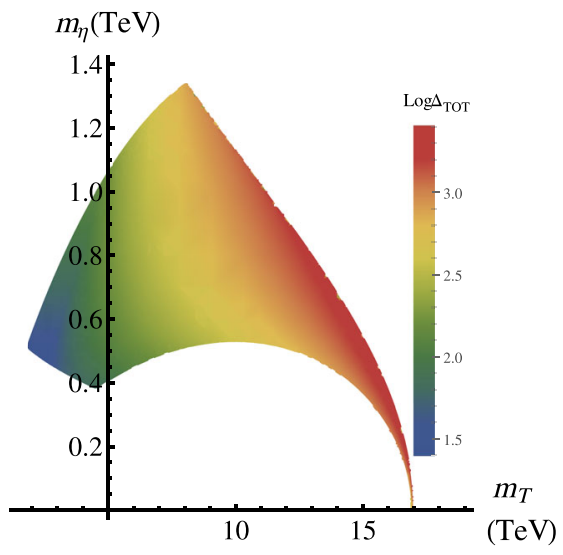

FIG. 1. Density plot of $\log \Delta_{\text {TOT }}$ in the $m_{\eta}-m_{T}$ plane for $f=8 \mathrm{TeV}$. Log means $\log _{10}$.

\section{Fermion mass diagonalization and flavor assumption}

Fermion mass diagonalization has been studied in Refs. [23,24]. In the lepton sector, the fermion mass matrices can be diagonalized by the following field rotations:

$$
\begin{gathered}
\left(\begin{array}{c}
N_{L n} \\
\nu_{L n}
\end{array}\right) \rightarrow\left(\begin{array}{cc}
c_{\delta} & s_{\delta} \\
s_{\delta} & -c_{\delta}
\end{array}\right)\left(\begin{array}{c}
N_{L n} \\
\nu_{L n}
\end{array}\right), \\
n=1,2,3, \quad \delta \equiv \frac{v}{\sqrt{2} f t_{\beta}}, \\
\left(\begin{array}{c}
e_{L} \\
\mu_{L} \\
\tau_{L}
\end{array}\right) \rightarrow U_{l}\left(\begin{array}{l}
e_{L} \\
\mu_{L} \\
\tau_{L}
\end{array}\right), \quad\left(\begin{array}{l}
e_{R} \\
\mu_{R} \\
\tau_{R}
\end{array}\right) \rightarrow W_{l}\left(\begin{array}{l}
e_{R} \\
\mu_{R} \\
\tau_{R}
\end{array}\right),
\end{gathered}
$$

where $U_{l}, W_{l}$ are both $3 \times 3$ unitary matrices. In this work, for simplicity we will assume that $U_{l}, W_{l}$ are both identity matrices. This leads to a simplification of some Feynman rules associated with the heavy neutrino $N$.

In the quark sector, first of all we perform field rotations in the right-handed sector as follows:

$u_{R 3}^{1}=\frac{-\lambda_{2}^{t} s_{\beta} t_{R}+\lambda_{1}^{t} c_{\beta} T_{R}}{\sqrt{\lambda_{1}^{t 2} c_{\beta}^{2}+\lambda_{2}^{t 2} s_{\beta}^{2}}}, \quad u_{R 3}^{2}=\frac{\lambda_{1}^{t} c_{\beta} t_{R}+\lambda_{2}^{t} s_{\beta} T_{R}}{\sqrt{\lambda_{1}^{t 2} c_{\beta}^{2}+\lambda_{2}^{t 2} s_{\beta}^{2}}}$,

$d_{R 1}^{1}=\frac{-\lambda_{2}^{d} s_{\beta} d_{R}+\lambda_{1}^{d} c_{\beta} D_{R}}{\sqrt{\lambda_{1}^{d 2} c_{\beta}^{2}+\lambda_{2}^{d 2} s_{\beta}^{2}}}, \quad d_{R 1}^{2}=\frac{\lambda_{1}^{d} c_{\beta} d_{R}+\lambda_{2}^{d} s_{\beta} D_{R}}{\sqrt{\lambda_{1}^{d 2} c_{\beta}^{2}+\lambda_{2}^{d 2} s_{\beta}^{2}}}$,

$d_{R 2}^{1}=\frac{-\lambda_{2}^{s} s_{\beta} s_{R}+\lambda_{1}^{s} c_{\beta} S_{R}}{\sqrt{\lambda_{1}^{s 2} c_{\beta}^{2}+\lambda_{2}^{s 2} s_{\beta}^{2}}}, \quad d_{R 2}^{2}=\frac{\lambda_{1}^{s} c_{\beta} s_{R}+\lambda_{2}^{s} s_{\beta} S_{R}}{\sqrt{\lambda_{1}^{s 2} c_{\beta}^{2}+\lambda_{2}^{s 2} s_{\beta}^{2}}}$.
For simplicity, the phenomenological studies done in this work will be carried out under the following flavor assumptions on the quark Yukawa Lagrangian (16):

$$
\begin{gathered}
\lambda_{u}^{T u}=\lambda_{u}^{T c}=\lambda_{u}^{12}=\lambda_{u}^{21}=\lambda_{u}^{31}=\lambda_{u}^{32}=0, \\
\lambda_{b}^{D}=\lambda_{b}^{S}=\lambda_{b}^{1}=\lambda_{b}^{2}=0 .
\end{gathered}
$$

These flavor assumptions turn off all of the generationcrossing quark flavor transitions and lead to a trivial Cabibbo-Kobayashi-Maskawa matrix, i.e., $V_{\mathrm{CKM}}=\mathbf{1}_{3 \times 3}$, which is not realistic. Nevertheless, in this paper we are concerned with the direct production of new physics particles at high-energy colliders rather than quark flavor observables. Also, for the parameter region that we are interested in, the phenomenology is not sensitive to the flavor assumptions adopted here, if the $\lambda$ 's in Eqs. (45) and (46), which characterize the generation-crossing quark flavor changing effects, are small.

With the above flavor assumptions, it is then straightforward to show that, up to $\mathcal{O}\left(\frac{v}{f}\right)$, after right-handed sector field rotations we only need to perform the following field rotations in the left-handed sector to diagonalize the quark mass matrices:

$$
\begin{gathered}
\left(\begin{array}{c}
t_{L} \\
T_{L}
\end{array}\right) \rightarrow\left(\begin{array}{cc}
1 & -\delta_{t} \\
\delta_{t} & 1
\end{array}\right)\left(\begin{array}{c}
t_{L} \\
T_{L}
\end{array}\right), \\
\left(\begin{array}{c}
d_{L} \\
D_{L}
\end{array}\right) \rightarrow\left(\begin{array}{cc}
1 & -\delta_{D d} \\
\delta_{D d} & 1
\end{array}\right)\left(\begin{array}{c}
d_{L} \\
D_{L}
\end{array}\right), \\
\left(\begin{array}{c}
s_{L} \\
S_{L}
\end{array}\right) \rightarrow\left(\begin{array}{cc}
1 & -\delta_{S s} \\
\delta_{S s} & 1
\end{array}\right)\left(\begin{array}{c}
s_{L} \\
S_{L}
\end{array}\right) .
\end{gathered}
$$

In the above equations, the field rotation parameters $\delta_{t}, \delta_{D d}$, $\delta_{S s}$ can be expressed using $f, \beta$ and the corresponding heavy fermion mass as follows ${ }^{6}$ :

$$
\begin{aligned}
& \delta_{t}=\frac{v}{2 \sqrt{2} f s_{\beta} c_{\beta}}\left(s_{\beta}^{2}-c_{\beta}^{2} \pm \sqrt{1-8 \frac{m_{t}^{2}}{v^{2}} \frac{f^{2}}{M_{T}^{2}} s_{\beta}^{2} c_{\beta}^{2}}\right), \\
& \delta_{D d}=-\frac{v}{2 \sqrt{2} f s_{\beta} c_{\beta}}\left(s_{\beta}^{2}-c_{\beta}^{2} \pm \sqrt{1-8 \frac{m_{d}^{2} f^{2}}{v^{2}} \frac{M_{D}^{2}}{M_{\beta}^{2}} c_{\beta}^{2}}\right),
\end{aligned}
$$

$$
\delta_{S s}=-\frac{v}{2 \sqrt{2} f s_{\beta} c_{\beta}}\left(s_{\beta}^{2}-c_{\beta}^{2} \pm \sqrt{1-8 \frac{m_{s}^{2}}{v^{2}} \frac{f^{2}}{M_{S}^{2}} s_{\beta}^{2} c_{\beta}^{2}}\right) .
$$

\footnotetext{
${ }^{6}$ Our expressions for $\delta_{t}, \delta_{D d}, \delta_{S s}$ differ from the corresponding expressions in Eq. (2.63) of Ref. [23]. The expressions of $\delta_{t}, \delta_{D d}$, $\delta_{S s}$ given in Ref. [23] are not consistent with their counterparts in Ref. [24]. Our calculation agrees with Ref. [24].
} 
Note that in the above equations, before the square root, both the plus sign and minus sign give possible solutions, which leads to a total of eight sign combinations. When we refer to the sign combination in these equations, we will list them according to the order $\delta_{t}, \delta_{D d}, \delta_{S s}$, as, e.g., $(+,+,+)$, $(+,+,-)$, etc., $m_{d}, m_{s}, M_{D}, M_{S}$ correspond to the mass of $d, s, D, S$, respectively. In the following we will simply neglect the small $m_{d}, m_{s}$; then, the expressions of $\delta_{D d}, \delta_{S s}$ become identical, apart from a possible sign difference before the square root. Then, we obtain the simple expression

$$
\delta_{D d}^{+}=\delta_{S s}^{+}=-\frac{v t_{\beta}}{\sqrt{2} f}, \quad \delta_{D d}^{-}=\delta_{S s}^{-}=\frac{v}{\sqrt{2} f t_{\beta}},
$$

where the superscripts indicate the sign choice for the corresponding rotation parameter. The rotation parameters $\delta_{t}, \delta_{D d}, \delta_{S s}$ are important since they appear directly in the coefficients of various interaction vertices which affect the $\eta$ phenomenology, as we will see.

\section{Lagrangian in the mass basis}

We are now prepared to present the Lagrangian in the mass basis which is relevant for the investigation of $\eta$ phenomenology. However, let us first note that there is a subtle issue regarding the diagonalization in the bosonic sector. After EWSB, it can be shown that the $C P$-odd sector scalar kinetic matrix in terms of the $\eta, \zeta, \chi, \omega$ fields is not canonically normalized. Also, there exist "unexpected" two-point vector-scalar transition terms like $Z^{\mu} \partial_{\mu} \eta$ after expanding the covariant derivative terms of the scalar fields. Therefore, a further field rotation (including a proper gauge fixing) is needed to diagonalize the bosonic sector. This subtle issue had been overlooked for a long time in the literature, and was only remedied in a recent paper [22]. In Ref. [22], an expression for the fraction of mass eigenstate $\eta$ field contained in the $\eta, \zeta, \chi, \omega$ fields originally introduced in the parametrization (4)-(6) was obtained, valid to all orders in $\xi \equiv \frac{v}{f}$, as follows (we collect the four fraction values into a four-component column vector $\Upsilon$ ):

$$
\Upsilon=\left(\begin{array}{c}
c_{\gamma+\delta}^{-1} \\
-c_{\gamma+\delta}^{-1}\left(s_{\delta}^{2} t_{\beta}-s_{\gamma}^{2} t_{\beta}^{-1}\right) \\
\frac{v}{\sqrt{2} f} c_{\gamma+\delta}^{-1}\left(c_{2 \delta} t_{\beta}-c_{2 \gamma} t_{\beta}^{-1}\right) \\
\frac{1}{2} c_{\gamma+\delta}^{-1}\left(s_{2 \delta} t_{\beta}+s_{2 \gamma} t_{\beta}^{-1}\right)
\end{array}\right),
$$

where

$$
\gamma \equiv \frac{v t_{\beta}}{\sqrt{2} f}, \quad \delta \equiv \frac{v}{\sqrt{2} f t_{\beta}}
$$

The $\Upsilon$ vector is involved in the derivation of all $\eta$-related mass eigenstate vertices. In particular, from the expression of $\Upsilon$ we see that there is an $\mathcal{O}(\xi)$ component of thw mass eigenstate $\eta$ contained in $\chi$. This has the following consequences. If we parametrize the mass eigenstate $\mathrm{ZH} \eta$ vertex as

$$
\begin{aligned}
\mathcal{L}_{Z H \eta}= & c_{Z H \eta}^{a s} Z^{\mu}\left(\eta \partial_{\mu} H-H \partial_{\mu} \eta\right) \\
& +c_{Z H \eta}^{s} Z^{\mu}\left(\eta \partial_{\mu} H+H \partial_{\mu} \eta\right),
\end{aligned}
$$

where $c_{Z H \eta}^{a s}$ denotes the coefficient of the antisymmetric $Z H \eta$ vertex, and $c_{Z H \eta}^{s}$ denotes the coefficient of the symmetric $Z H \eta$ vertex, then it was shown in Ref. [22] that

$$
\begin{gathered}
c_{Z H \eta}^{a s}=-\frac{g}{4 \sqrt{2} c_{W}^{3} t_{2 \beta}} \xi^{3}+\mathcal{O}\left(\xi^{5}\right), \\
c_{Z H \eta}^{s}=\frac{g}{\sqrt{2} c_{W} t_{2 \beta}} \xi+\frac{g}{24 \sqrt{2} c_{W} s_{2 \beta}}\left[\frac{8}{s_{2 \beta} t_{2 \beta}}\right. \\
\left.+3 c_{2 \beta}\left(8+\frac{6}{c_{W}^{2}}-\frac{1}{c_{W}^{4}}\right)\right] \xi^{3}+\mathcal{O}\left(\xi^{5}\right) .
\end{gathered}
$$

We see that the antisymmetric $Z H \eta$ vertex only shows up from $\mathcal{O}\left(\xi^{3}\right)$, in contrast to the results presented in Refs. $[17,18]$ which claimed the existence of antisymmetric $\mathrm{ZH} \eta$ vertex at $\mathcal{O}(\xi)$ due to the lack of an appropriate diagonalization in the bosonic sector.

This subtle issue of diagonalization in the bosonic sector also has an impact on the $\eta$ coupling to fermions. For instance, if we consider the expansion of $\epsilon_{i j k} \Phi_{1}^{i} \Phi_{2}^{j}$, with the help of the expression for the $\Upsilon$ vector in Eq. (54), we could find the following result for the neutral component:

$$
\epsilon_{i j k} \Phi_{1}^{i} \Phi_{2}^{j} \supset-i f\left(\begin{array}{c}
0 \\
f s_{\beta} c_{\beta} s_{\gamma+\delta}+\frac{1}{\sqrt{2}} c_{\gamma+\delta} H \\
0
\end{array}\right) .
$$

An important message from this is that $\epsilon_{i j k} \Phi_{1}^{i} \Phi_{2}^{j}$ does not contain any fraction of the mass eigenstate $\eta$ field, to all orders in $\xi$. Therefore, from Eq. (12) we immediately conclude that $\eta$ does not couple to a pair of charged leptons to all orders in $\xi$. This point was overlooked in previous studies [21,31] which relied on $\eta \rightarrow \tau \tau$.

In the following, let us collect the other mass eigenstate vertices that are relevant for $\eta$ phenomenology, to the first nontrivial order in $\xi$. In the Yukawa sector, we have the following couplings of $H$ and $\eta$ to a pair of fermions. 
(1) $H$ and $\eta$ couplings to the lepton sector:

$$
\begin{aligned}
\mathcal{L}_{L Y} \supset & -\sum_{n=1}^{3} \frac{m_{l n}}{\sqrt{2} f s_{\beta} c_{\beta} t_{\gamma+\delta}} H \bar{l}_{R n} l_{L n}+\sum_{n=1}^{3} \frac{M_{N n}}{\sqrt{2} f t_{\beta}} H \bar{N}_{R n} \nu_{L n} \\
& -i \sum_{n=1}^{3} \frac{M_{N n}}{\sqrt{2} f t_{\beta}} c_{\gamma+\delta} \eta \bar{N}_{R n} N_{L n}-i \sum_{n=1}^{3} \frac{M_{N n}}{\sqrt{2} f t_{\beta}} s_{\gamma+\delta} \eta \bar{N}_{R n} \nu_{L n}+\text { H.c. }
\end{aligned}
$$

(2) $H$ and $\eta$ couplings to the up-type quark sector:

$$
\begin{aligned}
\mathcal{L}_{Q Y} \supset & -\frac{m_{u}}{v} H \bar{u}_{R} u_{L}-\frac{m_{c}}{v} H \bar{c}_{R} c_{L} \\
& -\frac{m_{t}}{v} H \bar{t}_{R} t_{L}+\frac{m_{t}}{v}\left(\frac{\sqrt{2} v}{f t_{2 \beta}}+\delta_{t}\right) H \bar{t}_{R} T_{L}+\frac{M_{T}}{v} \delta_{t} H \bar{T}_{R} t_{L}+\frac{m_{t}^{2}}{v M_{T}} H \bar{T}_{R} T_{L} \\
& -i \frac{m_{t}}{v} \delta_{t} \eta \bar{t}_{R} t_{L}-i \frac{m_{t}}{v} \eta \bar{t}_{R} T_{L}+i \frac{M_{T}}{v}\left(\frac{v^{2}}{2 f^{2}}+\delta_{t}^{2}\right) \eta \bar{T}_{R} t_{L}+i \frac{M_{T}}{v} \delta_{t} \eta \bar{T}_{R} T_{L}+\text { H.c. }
\end{aligned}
$$

(3) $H$ and $\eta$ couplings to the down-type quark sector:

$$
\begin{aligned}
\mathcal{L}_{Q Y} \supset & -\frac{m_{b}}{v} H \bar{b}_{R} b_{L} \\
& -\frac{m_{d}}{v} H \bar{d}_{R} d_{L}+\frac{m_{d}}{v}\left(-\frac{\sqrt{2} v}{f t_{2 \beta}}+\delta_{D d}\right) H \bar{d}_{R} D_{L}+\frac{M_{D}}{v} \delta_{D d} H \bar{D}_{R} d_{L}+\frac{m_{d}^{2}}{v M_{D}} H \bar{D}_{R} D_{L} \\
& -\frac{m_{s}}{v} H \bar{s}_{R} s_{L}+\frac{m_{s}}{v}\left(-\frac{\sqrt{2} v}{f t_{2 \beta}}+\delta_{S s}\right) H \bar{s}_{R} S_{L}+\frac{M_{S}}{v} \delta_{S s} H \bar{S}_{R} s_{L}+\frac{m_{s}^{2}}{v M_{S}} H \bar{S}_{R} S_{L} \\
& -i \frac{m_{d}}{v} \delta_{D d} \eta \bar{d}_{R} d_{L}-i \frac{m_{d}}{v} \eta \bar{d}_{R} D_{L}+i \frac{M_{D}}{v}\left(\frac{v^{2}}{2 f^{2}}+\delta_{D d}^{2}\right) \eta \bar{D}_{R} d_{L}+i \frac{M_{D}}{v} \delta_{D d} \eta \bar{D}_{R} D_{L} \\
& -i \frac{m_{s}}{v} \delta_{S s} \eta \bar{s}_{R} s_{L}-i \frac{m_{s}}{v} \eta \bar{s}_{R} S_{L}+i \frac{M_{S}}{v}\left(\frac{v^{2}}{2 f^{2}}+\delta_{S s}^{2}\right) \eta \bar{S}_{R} s_{L}+i \frac{M_{S}}{v} \delta_{S s} \eta \bar{S}_{R} S_{L}+\text { H.c. }
\end{aligned}
$$

In the above equations, $m_{l n}, n=1,2,3$ denote the masses of $e, \mu, \tau$ leptons, $M_{N n}, n=1,2,3$ denote the masses of the three heavy neutral leptons $N_{n}$, and $m_{u}, m_{c}$ denote the masses of the $u, c$ quarks, respectively. $\eta$ can also be a decay product of the heavy fermions $N, T, D, S$, and therefore we also list the relevant Lagrangian for the heavy fermion gauge interaction which enters the heavy fermion decays,

$$
\begin{aligned}
\mathcal{L}_{\text {matter }} \supset & \frac{g v}{2 f t_{\beta}} W_{\mu}^{+} \bar{N}_{L m} \gamma^{\mu} l_{L m}-\frac{g v}{2 \sqrt{2} c_{W} f t_{\beta}} Z_{\mu} \bar{N}_{L m} \gamma^{\mu} \nu_{L m} \\
& -\frac{g \delta_{t}}{\sqrt{2}} W_{\mu}^{+} \bar{T}_{L} \gamma^{\mu} b_{L}-\frac{g \delta_{t}}{2 c_{W}} Z_{\mu} \bar{T}_{L} \gamma^{\mu} t_{L} \\
& -\frac{g \delta_{D d}}{\sqrt{2}} W_{\mu}^{+} \bar{u}_{L} \gamma^{\mu} D_{L}+\frac{g \delta_{D d}}{2 c_{W}} Z_{\mu} \bar{d}_{L} \gamma^{\mu} D_{L}-\frac{g \delta_{S s}}{\sqrt{2}} W_{\mu}^{+} \bar{c}_{L} \gamma^{\mu} S_{L}+\frac{g \delta_{S s}}{2 c_{W}} Z_{\mu} \bar{s}_{L} \gamma^{\mu} S_{L}+\text { H.c. }
\end{aligned}
$$

A further interesting possibility is that $\eta$ might come from the decay of a $Z^{\prime}$ boson. The $Z^{\prime}$-related parts of the interaction Lagrangian are listed below.

(1) $Z^{\prime}$ couplings to leptons:

$$
\begin{aligned}
\mathcal{L}_{\text {matter }} \supset & g \frac{1-t_{W}^{2}}{2 \sqrt{3-t_{W}^{2}}} \bar{l}_{L n} \gamma^{\mu} l_{L n} Z_{\mu}^{\prime}-g \frac{t_{W}^{2}}{\sqrt{3-t_{W}^{2}}} \bar{l}_{R n} \gamma^{\mu} l_{R n} Z_{\mu}^{\prime} \\
& +g \frac{1-t_{W}^{2}}{2 \sqrt{3-t_{W}^{2}}} \bar{\nu}_{L n} \gamma^{\mu} \nu_{L n} Z_{\mu}^{\prime}-g \frac{1}{\sqrt{3-t_{W}^{2}}} \bar{N}_{L n} \gamma^{\mu} N_{L n} Z_{\mu}^{\prime}
\end{aligned}
$$


(2) $Z^{\prime}$ couplings to third-generation quarks:

$$
\begin{aligned}
\mathcal{L}_{\text {matter }} \supset & -g \frac{3-2 t_{W}^{2}}{3 \sqrt{3-t_{W}^{2}}} \bar{T}_{L} \gamma^{\mu} T_{L} Z_{\mu}^{\prime}+g \frac{2 t_{W}^{2}}{3 \sqrt{3-t_{W}^{2}}} \bar{T}_{R} \gamma^{\mu} T_{R} Z_{\mu}^{\prime} \\
& +g \frac{3+t_{W}^{2}}{6 \sqrt{3-t_{W}^{2}}} \bar{t}_{L} \gamma^{\mu} t_{L} Z_{\mu}^{\prime}+g \frac{2 t_{W}^{2}}{3 \sqrt{3-t_{W}^{2}}} \bar{t}_{R} \gamma^{\mu} t_{R} Z_{\mu}^{\prime} \\
& +g \frac{3+t_{W}^{2}}{6 \sqrt{3-t_{W}^{2}}} \bar{b}_{L} \gamma^{\mu} b_{L} Z_{\mu}^{\prime}-g \frac{t_{W}^{2}}{3 \sqrt{3-t_{W}^{2}}} \bar{b}_{R} \gamma^{\mu} b_{R} Z_{\mu}^{\prime}
\end{aligned}
$$

(3) $Z^{\prime}$ couplings to first- and second-generation quarks:

$$
\begin{aligned}
\mathcal{L}_{\text {matter }} \supset & g \frac{\sqrt{3-t_{W}^{2}}}{3} \bar{D}_{L} \gamma^{\mu} D_{L} Z_{\mu}^{\prime}-g \frac{t_{W}^{2}}{3 \sqrt{3-t_{W}^{2}}} \bar{D}_{R} \gamma^{\mu} D_{R} Z_{\mu}^{\prime} \\
& -g \frac{\sqrt{3-t_{W}^{2}}}{6} \bar{d}_{L} \gamma^{\mu} d_{L} Z_{\mu}^{\prime}-g \frac{t_{W}^{2}}{3 \sqrt{3-t_{W}^{2}}} \bar{d}_{R} \gamma^{\mu} d_{R} Z_{\mu}^{\prime} \\
& -g \frac{\sqrt{3-t_{W}^{2}}}{6} \bar{u}_{L} \gamma^{\mu} u_{L} Z_{\mu}^{\prime}+g \frac{2 t_{W}^{2}}{3 \sqrt{3-t_{W}^{2}}} \bar{u}_{R} \gamma^{\mu} u_{R} Z_{\mu}^{\prime} \\
& + \text { terms with } \quad u \rightarrow c, d \rightarrow s, D \rightarrow S .
\end{aligned}
$$

(4) $Z^{\prime}$ couplings to bosons (relevant for $Z^{\prime}$ decay):

$$
\begin{aligned}
\mathcal{L}_{\text {gauge }} \supset & -i g \sqrt{3-t_{W}^{2}}\left(1-t_{W}^{2}\right) \frac{v^{2}}{8 f^{2}}\left\{\left(\partial_{\mu} Z_{\nu}^{\prime}\right)\left(W^{-\mu} W^{+\nu}-W^{+\mu} W^{-\nu}\right)\right. \\
& \left.+Z^{\prime \mu}\left[\left(\partial_{\mu} W_{\nu}^{+}\right) W^{-\nu}-\left(\partial_{\mu} W_{\nu}^{-}\right) W^{+\nu}\right]+Z^{\prime \nu}\left[\left(\partial_{\mu} W_{\nu}^{-}\right) W^{+\mu}-\left(\partial_{\mu} W_{\nu}^{+}\right) W^{-\mu}\right]\right\} \\
\mathcal{L}_{g k} \supset & -\frac{\sqrt{2} g v}{\sqrt{3-t_{W}^{2}} f t_{2 \beta}} Z^{\prime \mu}\left(\eta \partial_{\mu} H-H \partial_{\mu} \eta\right)-\frac{\sqrt{3-t_{W}^{2}} g v}{\sqrt{2} f t_{2 \beta}} Z^{\prime \mu}\left(\eta \partial_{\mu} H+H \partial_{\mu} \eta\right) \\
& -\frac{g^{2} v}{2 c_{W}^{2} \sqrt{3-t_{W}^{2}}} \eta Z^{\prime \mu} \frac{\left(Y_{\mu}^{0^{\dagger}}+Y_{\mu}^{0}\right)}{\sqrt{2}}+\frac{g^{2} v c_{2 W}}{2 c_{W}^{3} \sqrt{3-t_{W}^{2}}} H Z^{\prime \mu} Z_{\mu} .
\end{aligned}
$$

\section{SYMMETRIC VSS VERTICES}

In the derivation of the SLH Lagrangian in the mass basis we obtained the $\mathrm{ZH \eta}$ vertex in the form of Eq. (56), which contains two parts: the antisymmetric part $\left[Z^{\mu}\left(\eta \partial_{\mu} H-H \partial_{\mu} \eta\right)\right]$ and the symmetric part $\left[Z^{\mu}\left(\eta \partial_{\mu} H+\right.\right.$ $\left.\left.H \partial_{\mu} \eta\right)\right]^{7}$ An antisymmetric VSS vertex often appears in models based on a linearly realized scalar sector, such as the usual two-Higgs-doublet model (2HDM). It is natural to ask whether the symmetric VSS vertices can have any

\footnotetext{
${ }^{7}$ The Hermiticity requirement on the Lagrangian does not forbid the symmetric part. $Z_{\mu}, H, \eta$ are all real fields. $\partial_{\mu}$ does not lead to an additional minus sign under Hermitian conjugation because in quantum field theory $x^{\mu}$ 's are labels, not operators. This is not to be confused with the situation in ordinary quantum mechanics.
}

physical effect. We note that in a Lorentz-invariant $\mathrm{ZH} \eta$ vertex, the $\partial_{\mu}$ may act on any of the three fields $\left(Z^{\mu}, H, \eta\right)$. However because a total derivative term $\partial_{\mu}\left(Z^{\mu} H \eta\right)$ has no physical effects, we therefore expect at most two independent contributions from the interaction of one vector field with two scalar fields. If symmetric VSS vertices are allowed and present in a general theory and could lead to distinct physical effects, it would mean that a vector field could interact with two scalar fields in a manner different from the usually expected antisymmetric pattern, which may further reveal interesting features of the enlarged scalar sector.

Let us first note that the symmetric VSS Lagrangian $Z^{\mu}\left(\eta \partial_{\mu} H+H \partial_{\mu} \eta\right)$ can be written as

$$
Z_{\mu} \partial^{\mu}(H \eta)
$$


via Leibniz's rule and is therefore (via integration by parts) equivalent to

$$
-\left(\partial^{\mu} Z_{\mu}\right)(H \eta)
$$

in the Lagrangian formulation of the theory. A reflective reader might at this moment wonder whether terms like Eq. (70) indeed contribute to $S$-matrix elements if canonical quantization is adopted. Note that what matters in canonical quantization is the interaction Hamiltonian in the interaction picture (denoted $H_{I}^{\mathrm{int}}$ ), and if $Z^{\mu}$ is a massive spin-1 field, then the corresponding interaction picture field operator $Z_{I}^{\mu}$ (the subscript " $I$ " denotes interaction picture) will automatically satisfy [32]

$$
\partial_{\mu} Z_{I}^{\mu}=0 .
$$

It is tempting to arrive at the conclusion that terms like Eq. (70) cannot contribute to $S$-matrix elements due to Eq. (71). Actually, this is not quite correct. The correct procedure to go from the classical Lagrangian to the interaction Hamiltonian in the interaction picture $H_{I}^{\text {int }}$ is to (i) identify appropriate canonical coordinates and their conjugate momenta, (ii) perform a Legendre transformation to obtain the Hamiltonian and express it in terms of canonical coordinates and their conjugate momenta, (iii) promote the canonical variables to field operators satisfying appropriate canonical commutation relations, and finally (iv) split the Hamiltonian into a free part and an interaction part and replace the Heisenberg-picture quantities with their interaction-picture counterparts [32]. If this procedure is strictly followed, we would find that only the spatial components of $Z^{\mu}$ can be treated as independent canonical coordinates while $Z^{0}$ is dependent because regardless of whether we start with Eq. (69) or Eq. (70) the derivative of the Lagrangian with respect to $\dot{Z}_{0}$ cannot be made to satisfy canonical commutation relations. To avoid the appearance of $\partial^{0} Z_{0}$ in the Hamiltonian we could start with Eq. (69), and then the problem turns out to be the same as that treated in Sec. 7.5 of Ref. [32]. Using the results there, we could see that Eq. (69) leads to a term

$$
-Z_{I}^{\mu} \partial_{\mu}\left(h_{I} A_{I}\right)
$$

in the interaction Hamiltonian in the interaction picture (barring a Lorentz noncovariant term which is not shown here). This will certainly lead to a vertex Feynman rule

$$
-k^{\mu}
$$

where $k^{\mu}$ is the $Z$ momentum flowing into the vertex. This vertex Feynman rule could also be derived from Eq. (69) via the path-integral method. Notice that it is not legitimate to perform integration by parts in the interaction-picture Hamiltonian $H_{I}^{\text {int }}$ to obtain

$$
\left(\partial_{\mu} Z_{I}^{\mu}\right)\left(h_{I} A_{I}\right)
$$

from Eq. (72). ${ }^{8}$

The appearance of $\partial_{\mu} Z^{\mu}$ in Eq. (70) is reminiscent of covariant gauge fixing in gauge field theories. Equation (70) is not gauge invariant; nevertheless, at this moment let us suppose that it can be deduced from a gaugeinvariant operator. Because we are dealing with quantum field theories it is important not to confuse them with classical field theories. In a classical gauge field theory a gauge-fixing condition (such as the Landau gauge condition $\partial_{\mu} Z^{\mu}=0$ ) is employed so that the solutions of the equation of motion are required to also satisfy the gaugefixing condition. In quantum field theory all classical field configurations, regardless of whether they satisfy the classical equation of motion, are to be integrated over in the path integral. The usually adopted covariant gauge, the general $R_{\xi}$ gauge, actually corresponds to a Gaussian smearing of a class of covariant gauge conditions and does not strictly force the classical field to satisfy a simple gauge-fixing equation. However, the limit $\xi \rightarrow 0$ makes the gauge-fixing functional act like a delta function imposing the Landau gauge condition $\partial_{\mu} Z^{\mu}=0$ [32]. Therefore, it is heuristic to guess that in the Landau gauge, symmetric VSS vertices do not contribute to the $S$-matrix of the theory. However, we should not forget that in the Landau gauge it is necessary to take into account the Goldstone contribution to the $S$-matrix, and also the associated ghost contribution when we go beyond tree level in perturbation theory. This observation suggests that at tree level, processes involving symmetric VSS vertices can be seen as purely Goldstone mediated.

Physical effects of antisymmetric VSS vertices have been well studied in the literature. For example, in the 2HDM, a benchmark process which embodies the effect of antisymmetric VSS vertices is

$$
f+\bar{f} \rightarrow A+h
$$

where $A$ and $h$ denote a generic $C P$-odd and $C P$-even 2HDM Higgs boson, respectively. The corresponding Feynman diagram is shown in Fig. 2 in unitarity gauge. Now suppose we replace the antisymmetric VSS $Z h A$ vertex in Fig. 2 by a completely symmetric VSS ZhA vertex. It is obvious that if the $Z$ boson is on shell, then the amplitude should vanish since for an on-shell massive vector boson we have the relation $p \cdot \epsilon=0$ for its

\footnotetext{
${ }^{8}$ More specifically, integration by parts for spatial components of $H_{I}^{\text {int }}$ should be fine if the fields are assumed to satisfy certain boundary conditions, which is usually the case. However, integration by parts for the temporal component of $H_{I}^{\text {int }}$ is problematic since in the expression for the scattering operator $S=T \exp \left(-i \int_{-\infty}^{+\infty} H_{I}^{\mathrm{int}} d t\right)$ the temporal integration is actually twisted by the time ordering. No such problem exists if we adopt the path-integral method.
} 


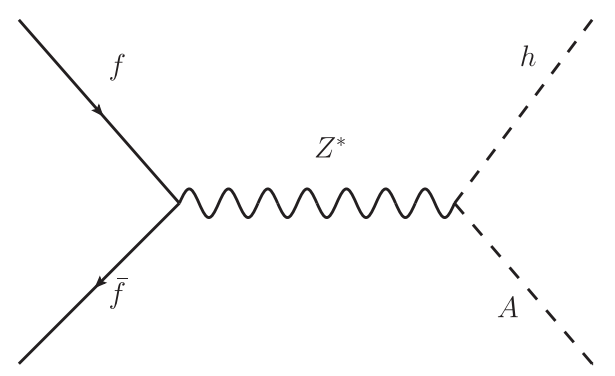

FIG. 2. Associated production of $h$ and $A$.

momentum and polarization vectors. It is tempting to proceed with the case where the $Z$ boson is off shell. The amplitude in this case can be examined from two perspectives. First, we can perform the calculation in unitarity gauge. In this gauge, the result of dotting the $Z$ momentum $p$ at the $Z h A$ vertex into its $s$-channel propagator is again proportional to the $Z$ momentum $p$ at the $Z f \bar{f}$ vertex. It is then obvious that only the axial-vector part of the $Z f \bar{f}$ vertex contributes to the amplitude, with a contribution proportional to the fermion mass $m_{f}$. Alternatively, we may perform the calculation in Landau gauge $(\xi=0)$, in which the diagram shown in Fig. 2 does not contribute to the amplitude; nevertheless, we need to take into account the $s$-channel Goldstone-mediated amplitude, which again gives a contribution proportional to the fermion mass $m_{f}$.

Although usually $f$ is a light fermion with negligible mass effects, we might be interested in the case where $f$ is heavy with important mass effects, e.g., the top quark. If in this case the symmetric VSS vertex could lead to physical effects, we would seem to produce a paradox in the SLH. In the SLH there exists a symmetric $Z H \eta$ vertex; however, if we consider a linearly realized SLH as a UV completion, then it cannot lead to symmetric VSS vertices and hence there will be no related physical effects. Since the usual nonlinearly realized SLH can be related to a linearly realized SLH via an appropriate field redefinition, the above discussion seems to violate the field redefinition invariance of the $S$-matrix element. ${ }^{9}$ We can turn the argument around to use the field redefinition invariance to infer the existence of an additional contribution in the SLH which also contributes to the $f \bar{f} \rightarrow H \eta$ process such that the field redefinition invariance is maintained. In fact, if we examine the Yukawa part of the SLH Lagrangian, we would find the following four-point contact vertex $\left(m_{f}\right.$ denotes the mass of $f$ ):

$$
\mathcal{L} \supset i \frac{2 \sqrt{2} g_{A}}{f t_{2 \beta}} \frac{m_{f}}{v} H \eta \bar{f} \gamma^{5} f
$$

\footnotetext{
${ }^{9}$ The radial mode does not help since it does not have the required $C P$ property.
}

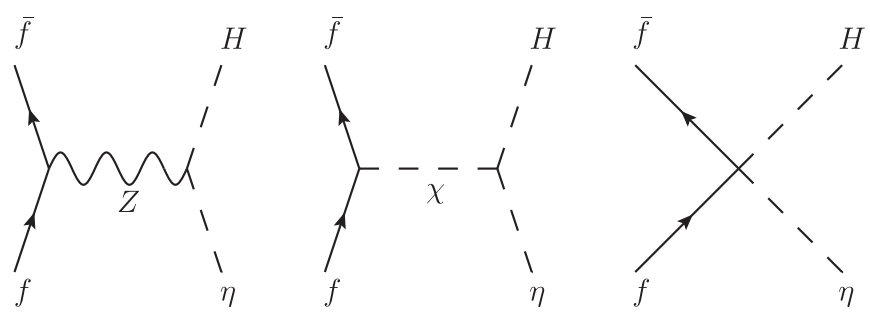

FIG. 3. Feynman diagrams in the SLH for $f \bar{f} \rightarrow H \eta$ in $R_{\xi}$ gauge.

Here $g_{A}$ is the axial coupling of the fermion $f$ which also appears in its interaction with the $Z$ boson and the associated Goldstone $\chi$ as

$$
\mathcal{L} \supset \frac{g}{2 c_{W}} Z^{\mu} \bar{f} \gamma_{\mu}\left(g_{V}+g_{A} \gamma^{5}\right) f+i \frac{2 g_{A} m_{f}}{v} \bar{f} \gamma^{5} f \chi .
$$

Now if we compute the amplitude for $f \bar{f} \rightarrow H \eta$ in $R_{\xi}$ gauge, we need to include three contributions: $s$-channel $Z$ exchange, $s$-channel $\chi$ exchange, and the $\mathrm{ffH} \eta$ contact interaction, as shown in Fig. 3. The amplitudes corresponding to these three diagrams are computed to be (from left to right)

$$
\begin{gathered}
i \mathcal{M}_{I}=\frac{\sqrt{2}}{v f t_{2 \beta}} \frac{-\xi m_{Z}^{2}}{q^{2}-\xi m_{Z}^{2}} 2 g_{A} m_{f} \bar{v}\left(p_{\bar{f}}\right) \gamma^{5} u\left(p_{f}\right), \\
i \mathcal{M}_{I I}=\frac{\sqrt{2}}{v f t_{2 \beta}} \frac{q^{2}}{q^{2}-\xi m_{Z}^{2}} 2 g_{A} m_{f} \bar{v}\left(p_{\bar{f}}\right) \gamma^{5} u\left(p_{f}\right), \\
i \mathcal{M}_{I I I}=-\frac{\sqrt{2}}{v f t_{2 \beta}} 2 g_{A} m_{f} \bar{v}\left(p_{\bar{f}}\right) \gamma^{5} u\left(p_{f}\right) .
\end{gathered}
$$

Here $p_{f}$ and $p_{\bar{f}}$ are the four-momenta of $f$ and $\bar{f}$, respectively and $q \equiv p_{f}+p_{\bar{f}}$. When we add the three contributions, we find

$$
i \mathcal{M}_{I}+i \mathcal{M}_{I I}+i \mathcal{M}_{I I I}=0
$$

which is exactly what we would expect from field redefinition invariance. Moreover, we see that the $Z$ and $\chi$ contributions add up to be gauge independent, while the contact interaction contribution itself is gauge independent.

Here we would like to mention a further subtle point related to the symmetric VSS vertex. It might still be somewhat counterintuitive that the contribution from the symmetric $Z H \eta$ vertex is canceled by the contribution from the $\mathrm{ffH} \eta$ contact vertex, since the former contribution should know the position of the $Z$ pole and therefore vanish for an on-shell $Z$ boson, while the latter certainly does not "feel" the $Z$ pole. To illustrate this issue, we can include the effect of the $Z$ boson width $\Gamma_{Z}$ so that the $Z$ boson propagator in the unitarity gauge is written as 


$$
\frac{-g^{\mu \nu}+\frac{q^{\mu} q^{\nu}}{m_{Z}^{2}}}{q^{2}-m_{Z}^{2}+i m_{Z} \Gamma_{Z}} .
$$

When this propagator is dotted into $q_{\nu}$ coming from the symmetric VSS Feynman rule, at $q^{2}=m_{Z}^{2}$ it will vanish, which seems quite plausible given our previous argument that a symmetric VSS vertex does not contribute to the process in which the related vector boson is on shell. However, this immediately leads to the paradoxical situation that near the on-shell region the field redefinition invariance is again violated since the contribution from the $f f H \eta$ contact vertex certainly does not know about the $Z$ pole.

The resolution of this paradox consists in the treatment of the particle width in its propagator. The naive treatment in Eq. (82) is actually not quite correct and will in general lead to results that violate the Ward-Takahashi identities. A proper treatment can be made by, e.g., employing the complex mass scheme which properly retains gauge invariance. The final result is that, of course, no exotic structure appears near the $Z$ pole and the field redefinition invariance is maintained.

\section{CONSTRAINTS FROM ELECTROWEAK PRECISION OBSERVABLES}

As discussed in Sec. II, in the study of the pseudoaxion phenomenology there are eight sign combinations for the rotation parameters $\delta_{t}, \delta_{D d}, \delta_{S s}$. Moreover, when the lepton sector is relevant, either $t_{\beta} \geq 1$ or $t_{\beta}<1$ could be possible, leading to further complications. Nevertheless, as will be shown in this section, the number of possibilities greatly reduces if we require the following.

(1) The parameter space under consideration is favored by naturalness considerations and thus embodies (to some extent) the original motivation of the SLH model.

(2) The parameter space under consideration is allowed by electroweak precision measurements.

As discussed in Sec. II, the first requirement points to the region characterized by a small top partner mass. In the SLH, currently the lower bound on the top partner mass is derived from Eq. (36) where $f$ is stringently constrained by dilepton resonance searches. Constraints from direct searches for top partner production are not as competitive at the moment. For given $f$, a small top partner mass could be obtained by requiring a large $t_{\beta}\left(\right.$ or $t_{\beta}^{-1}$ for $t_{\beta}<1$ ), which is in turn bounded by unitarity considerations. To summarize, the first requirement points to the region characterized by a small $f$ and large $t_{\beta}$ (or $t_{\beta}^{-1}$ for $t_{\beta}<1$ ).

As for the second requirement, in the present work we consider the following electroweak observables.

(1) The $W$ boson mass $m_{W}$.

(2) $R$ observables measured at the $Z$ pole, $R_{b}, R_{c}, R_{e}$, $R_{\mu}, R_{\tau}$, which are defined by

$$
\begin{aligned}
R_{b} & \equiv \Gamma(b \bar{b}) / \Gamma(\mathrm{had}), & R_{c} & \equiv \Gamma(c \bar{c}) / \Gamma(\mathrm{had}), \\
R_{l} & \equiv \Gamma(\mathrm{had}) / \Gamma\left(l^{+} l^{-}\right), & l & =e, \mu, \tau,
\end{aligned}
$$

in which $\Gamma$ (had) denotes the total hadronic width of the $Z$ boson, and $\Gamma(b \bar{b}), \Gamma(c \bar{c}), \Gamma\left(l^{+} l^{-}\right)$denote the $Z$-boson partial widths into $b \bar{b}, c \bar{c}, l^{+} l^{-}$channels. To set up the calculation we choose the fine-structure constant $\alpha_{\mathrm{em}} \equiv \frac{e^{2}}{4 \pi}$ (defined at the $Z$ pole), the Fermi constant $G_{F}$, and $Z$ boson mass $m_{Z}$ as the input parameters. Expressed with the SM quantities, we have the tree-level relations

$$
\begin{gathered}
e=g_{\mathrm{SM}} s_{W, \mathrm{SM}}, \quad \frac{G_{F}}{\sqrt{2}}=\frac{g_{\mathrm{SM}}^{2}}{8 m_{W, \mathrm{SM}}^{2}}, \\
m_{Z}^{2}=\frac{g_{\mathrm{SM}}^{2} v_{\mathrm{SM}}^{2}}{4 c_{W, \mathrm{SM}}^{2}}, \quad m_{W, \mathrm{SM}}^{2}=\frac{1}{4} g_{\mathrm{SM}}^{2} v_{\mathrm{SM}}^{2} .
\end{gathered}
$$

These relations are modified in the SLH to be

$$
e=g s_{W}, \quad \frac{G_{F}}{\sqrt{2}}=\frac{g^{2}}{8 m_{W, \mathrm{SLH}}^{2}}\left(1-\frac{v^{2}}{4 f^{2} t_{\beta}^{2}}\right)^{2},
$$

$$
\begin{array}{r}
m_{Z}^{2}=\frac{g^{2} v^{2}}{4 c_{W}^{2}}+\frac{g^{2}}{32 c_{W}^{2}}\left[c_{W}^{-2}\left(3-t_{W}^{2}\right)-\frac{4}{3} s_{\beta}^{-2} c_{\beta}^{-2}\right] \frac{v^{4}}{f^{2}}, \\
m_{W, \mathrm{SLH}}^{2}=\frac{1}{4} g^{2} v^{2}+\frac{1}{24} g^{2}\left(3-s_{\beta}^{-2} c_{\beta}^{-2}\right) \frac{v^{4}}{f^{2}} .
\end{array}
$$

Here we note that in the above equations, as in Sec. II, $g, v$, $s_{W}$ represent quantities in the SLH and are thus different from the SM quantities $g_{\mathrm{SM}}, v_{\mathrm{SM}}, s_{W, \mathrm{SM}}$. From the above two sets of relations we may derive

$$
\begin{aligned}
& \frac{m_{W, \mathrm{SLH}}^{2}}{m_{W, \mathrm{SM}}^{2}}=1+\frac{1}{8}\left(1-t_{W, \mathrm{SM}}^{2}+\frac{1-c_{W, \mathrm{SM}}^{2}}{2 c_{W, \mathrm{SM}}^{2}-1} \frac{4}{t_{\beta}^{2}}\right) \frac{v_{\mathrm{SM}}^{2}}{f^{2}}, \\
& \frac{s_{W}^{2}}{s_{W, \mathrm{SM}}^{2}}=1-\frac{1}{8}\left(1-t_{W, \mathrm{SM}}^{2}+\frac{c_{W, \mathrm{SM}}^{2}}{2 c_{W, \mathrm{SM}}^{2}-1} \frac{4}{t_{\beta}^{2}}\right) \frac{v_{\mathrm{SM}}^{2}}{f^{2}} .
\end{aligned}
$$

To calculate the $R$ observables in the SLH we also need the modified $Z$ couplings to light fermions. Although the corrections relative to the SM are of $\frac{v^{2}}{f^{2}}$ order, they are still relevant since the $R$ observables have been measured to a few per mille precision. In such a case the diagonal entries in the rotational matrices in Eq. (49) should be understood as $1-\frac{1}{2} \delta_{D d}^{2}$ and $1-\frac{1}{2} \delta_{S s}^{2}$, respectively. Then the modified $Z$ couplings to light fermions in the SLH can be written as 


$$
\begin{aligned}
g_{L, Z, f}^{\prime} & =g_{L, Z, f}+\delta_{Z} g_{L, Z^{\prime}, f}, \\
g_{R, Z, f}^{\prime} & =g_{R, Z, f}+\delta_{Z} g_{R, Z^{\prime}, f}, \\
\text { for } f & =u, c, b, e, \mu, \tau .
\end{aligned}
$$

In the above equations, $\delta_{Z}$ is the $\mathcal{O}\left(\frac{v^{2}}{f^{2}}\right) Z-Z^{\prime}$ mixing angle, appearing in the mixing relation

$$
Z^{\prime}=Z_{m}^{\prime}+\delta_{Z} Z_{m}, \quad Z=Z_{m}-\delta_{Z} Z_{m}^{\prime} .
$$

Here $Z_{m}, Z_{m}^{\prime}$ denote the final mass eigenstates after the $\mathcal{O}\left(\frac{v^{2}}{f^{2}}\right)$ rotation while $Z, Z^{\prime}$ denote the states before the $\mathcal{O}\left(\frac{v^{2}}{f^{2}}\right)$ rotation, as defined via Eq. (11). In the process of gauge boson mass diagonalization, $\delta_{Z}$ is computed to be

$$
\delta_{Z}=-\frac{\left(1-t_{W}^{2}\right) \sqrt{3-t_{W}^{2}}}{8 c_{W}} \frac{v^{2}}{f^{2}} .
$$

In Eq. (91), $g_{L, Z, f}=\frac{g}{c_{W}}\left(T_{3}^{f}-Q_{f} s_{W}^{2}\right), g_{R, Z, f}=-\frac{g}{c_{W}} Q_{f} s_{W}^{2}$ are leading-order coefficients of the Lagrangian terms $\bar{f}_{L} \gamma^{\mu} f_{L} Z_{\mu}, \bar{f}_{R} \gamma^{\mu} f_{R} Z_{\mu}$, and $T_{3}^{f}, Q_{f}$ denote the third components of the isospin and the electric charge of $f$, respectively. $g_{L, Z^{\prime}, f}, g_{R, Z^{\prime}, f}$ are leading-order coefficients of the Lagrangian terms $\bar{f}_{L} \gamma^{\mu} f_{L} Z_{\mu}^{\prime}, \bar{f}_{R} \gamma^{\mu} f_{R} Z_{\mu}^{\prime}$, which are given in Eqs. (64)-(66). $g_{L, Z, f}^{\prime}, g_{R, Z, f}^{\prime}$ in Eq. (91) denote the coefficients of the Lagrangian terms $\bar{f}_{L} \gamma^{\mu} f_{L} Z_{\mu}, \bar{f}_{R} \gamma^{\mu} f_{R} Z_{\mu}$ and $T_{3}^{f}, Q_{f}$, to $\mathcal{O}\left(\frac{v^{2}}{f^{2}}\right)$ precision.

For $f=d$ the modified $Z$ couplings in the SLH turn out to be

$$
\begin{aligned}
g_{L, Z, d}^{\prime} & =g_{L, Z, d}+\delta_{Z} g_{L, Z^{\prime}, d}+\delta_{D d}^{2}\left(g_{L, Z, D}-g_{L, Z, d}\right), \\
g_{R, Z, d}^{\prime} & =g_{R, Z, d}+\delta_{Z} g_{R, Z^{\prime}, d}
\end{aligned}
$$

Obviously the additional correction is due to the lefthanded $D-d$ mixing. The corresponding formulas for $f=s$ can be obtained by the replacements $d \rightarrow s, D \rightarrow S . g_{L, Z, D}$, $g_{L, Z, S}$ are leading-order coefficients of the Lagrangian terms $\bar{D}_{L} \gamma^{\mu} D_{L} Z_{\mu}, \bar{S}_{R} \gamma^{\mu} S_{R} Z_{\mu}$,

$$
g_{L, Z, D}=g_{L, Z, S}=\frac{1}{3} g s_{W} t_{W} .
$$

Now we have all of the SLH couplings that are necessary to calculate the $R$ observables. It should be noted that in the above coupling formulas, $s_{W}, c_{W}, t_{W}$ are quantities in the SLH and are therefore different from their SM counterparts $s_{W, \mathrm{SM}}, c_{W, \mathrm{SM}}, t_{W, \mathrm{SM}}$; see Eq. (90). Therefore, the modification of $Z$ couplings to light fermions relative to the $\mathrm{SM}$ is caused by three factors: $Z-Z^{\prime}$ mixing, left-handed $D-d, S-s$ mixing, and the correction of the weak-mixing angle.

A $95 \%$ C.L. constraint can be obtained in the $f-t_{\beta}$ plane by performing a $\chi^{2}$ fit of the five $R$ observables. The $\chi^{2}$ is defined by
TABLE I. Experimental values and the SM predictions of the $R$ observables.

\begin{tabular}{lcc}
\hline \hline Quantity & Value & Standard Model \\
\hline$R_{e}$ & $20.804 \pm 0.050$ & $20.737 \pm 0.010$ \\
$R_{\mu}$ & $20.785 \pm 0.033$ & $20.737 \pm 0.010$ \\
$R_{\tau}$ & $20.764 \pm 0.045$ & $20.782 \pm 0.010$ \\
$R_{b}$ & $0.21629 \pm 0.00066$ & $0.21582 \pm 0.00002$ \\
$R_{c}$ & $0.1721 \pm 0.0030$ & $0.17221 \pm 0.00003$ \\
\hline \hline
\end{tabular}

$$
\chi^{2}=\sum_{f=b, c, e, \mu, \tau} \frac{\left(R_{f, S L H}-R_{f}\right)^{2}}{\delta_{R_{f}}^{2}+\delta_{R_{f, S \mathrm{M}}}^{2}} .
$$

In the above equation, $R_{f}$ denotes the experimental values and $\delta_{R_{f}}$ denotes the associated experimental uncertainties. Also, $R_{f, \mathrm{SM}}$ is the SM theory prediction and $\delta_{R_{f, \mathrm{SM}}}$ denotes the associated theory uncertainty. Their values are listed in Table I [33]. As for the constraint from the $W$-boson mass, we treat it separately and consider two more precise measurements [33],

$$
\begin{gathered}
m_{W}=80.387 \pm 0.016 \mathrm{GeV} \quad \text { (Tevatron) } \\
m_{W}=80.370 \pm 0.019 \mathrm{GeV} \quad(\text { ATLAS) }
\end{gathered}
$$

while we note that the SM prediction for $m_{W}$ is [33]

$$
m_{W, \mathrm{SM}}=80.358 \pm 0.004 \mathrm{GeV} .
$$

In Fig. 4 the results of the electroweak precision analysis of $m_{W}$ and $R$ observables are shown. To clarify the situation we present the results according to whether $t_{\beta} \geq 1$ and the sign combination of the rotation parameters $\delta_{D d}, \delta_{S s}$ [see Eq. (53)]. At first sight there are eight possibilities in total; however, it is immediately recognized that $\delta_{D d}^{+}, \delta_{S s}^{-}$, and $\delta_{D d}^{-}, \delta_{S s}^{+}$make no difference in terms of constraints in the $f-t_{\beta}$ plane, reducing the number of possibilities to six. Therefore we obtain the six panels in Fig. 4, with each panel showing one possibility as described in the caption.

For all of the panels, the green and yellow regions correspond to parameter points that are allowed by the $\chi^{2}$ fit of $R$ observables at $68 \%$ and $95 \%$ C.L., respectively. These allowed regions do not exhibit a $t_{\beta} \rightarrow t_{\beta}^{-1}$ symmetry (e.g., the allowed regions in the upper right panel and the lower left panel still differ under the transformation $t_{\beta} \rightarrow t_{\beta}^{-1}$ ), since in the computation of $R$ observables, the correction of $s_{W}^{2}$ relative to its SM value has to be taken into account, as was pointed out previously. When $f$ is larger than about $17 \mathrm{TeV}$ there will be a lower theoretical bound (from the mass relation) on $t_{\beta}$ or $t_{\beta}^{-1}$ which is larger than 1 , corresponding to the white region at large $f$ and small $t_{\beta}$ or $t_{\beta}^{-1}$ in each panel. The $2 \sigma$ constraints from $m_{W}$ measurements are simply implemented by requiring 

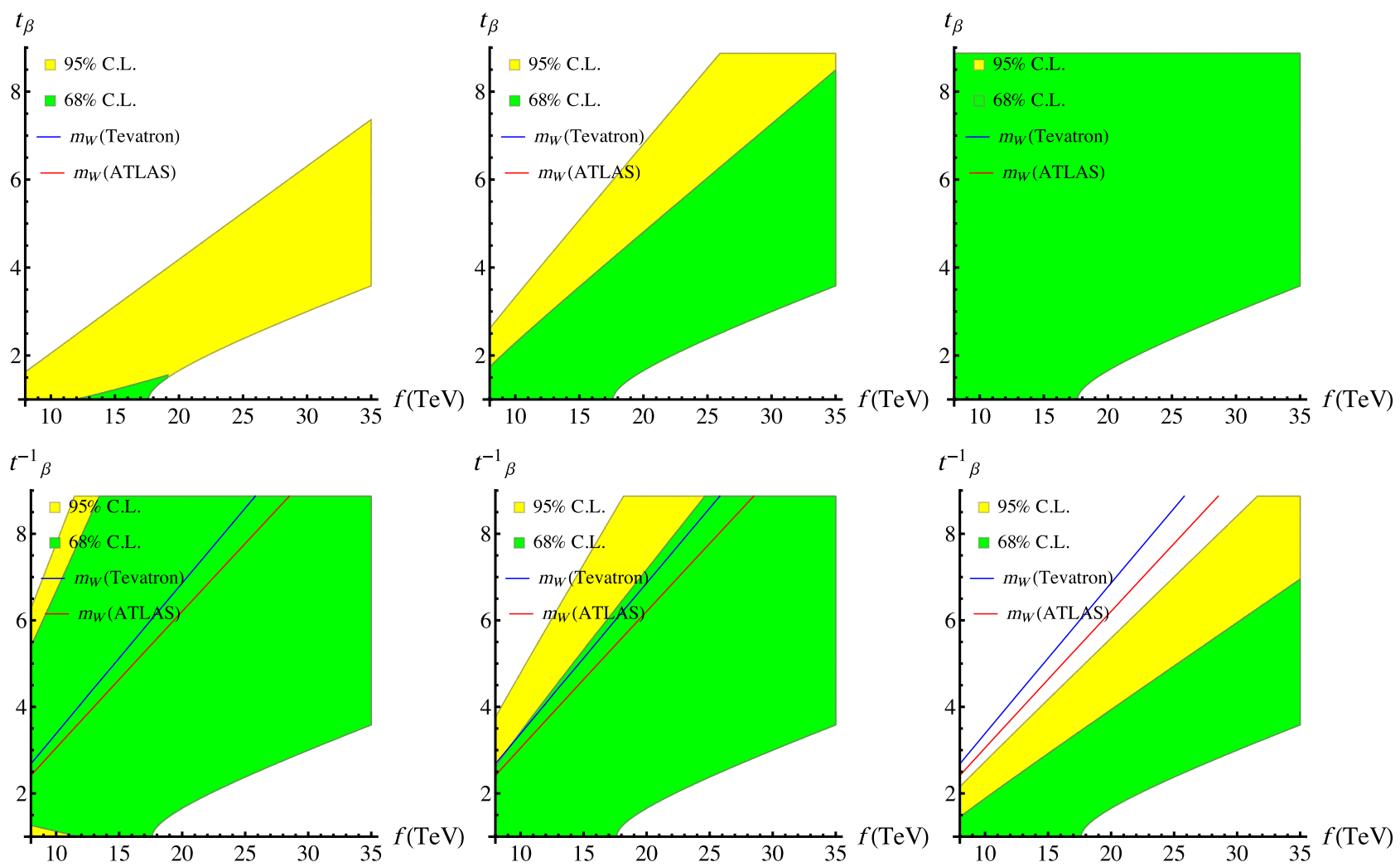

FIG. 4. Constraints from $m_{W}$ and $R$ observables in the $f-t_{\beta}$ plane. Upper left: $t_{\beta} \geq 1, \delta_{D d}^{+}, \delta_{S s}^{+}$; upper middle: $t_{\beta} \geq 1, \delta_{D d}^{+}, \delta_{S s}^{-}$or $t_{\beta} \geq 1$, $\delta_{D d}^{-}, \delta_{S s}^{+}$; upper right: $t_{\beta} \geq 1, \delta_{D d}^{-}, \delta_{S s}^{-}$; lower left: $t_{\beta} \leq 1, \delta_{D d}^{+}, \delta_{S s}^{+}$; lower middle: $t_{\beta} \leq 1, \delta_{D d}^{+}, \delta_{S s}^{-}$or $t_{\beta} \geq 1, \delta_{D d}^{-}, \delta_{S s}^{+}$; lower right: $t_{\beta} \leq 1$, $\delta_{D d}^{-}, \delta_{S s}^{-}$. See the text for a detailed description.

$$
\left|m_{W, S L H}-m_{W}\right|<2 \sqrt{\delta_{m_{W}}^{2}+\delta_{m_{W, S M}}^{2}} \cdot
$$

In the above equation $m_{W}$ denotes the experimentally measured $W$-boson mass and $\delta_{m_{W}}$ and $\delta_{m_{W, S M}}$ denote the associated experimental and theoretical uncertainties, respectively. We superimpose the constraint boundary on the six plots as blue or red lines, representing constraints from Tevatron or ATLAS measurements, respectively. For all of these $m_{W}$ constraint boundary lines, the regions on the right side of the lines are allowed at the $2 \sigma$ level.

As can be seen from Fig. 4, if $t_{\beta}<1$, then the region favored by naturalness consideration is disfavored by constraints from both $R$ observables and $W$-boson mass measurements, regardless of the sign combination of the rotation parameters $\delta_{D d}, \delta_{S s}$. If $t_{\beta} \geq 1$, then $W$-boson mass measurements do not constrain the parameter region favored by naturalness consideration. However, in this case constraints from $R$ observables are significant when any of the rotation parameters $\delta_{D d}, \delta_{S s}$ adopt the plus sign in Eq. (53). This is because a plus sign leads to a large $t_{\beta}$ enhancement of the rotation parameter and therefore a larger deviation of $Z$ couplings to the corresponding fermion. Although the lower bound on $f$ has been pushed to around $7.5 \mathrm{TeV}$ by LHC dilepton resonance searches, the $R$ observable constraints still force us to avoid this $t_{\beta}$ enhancement, and consequently the only possibility left is $\delta_{D d}^{-}, \delta_{S s}^{-}$with $t_{\beta} \geq 1$. This result has important consequences for the pseudoaxion phenomenology since the sign combinations of $\delta_{D d}, \delta_{S s}$ will determine how $\eta$ interacts with the $D, S$ quarks which in turn influences the decay and production of the $\eta$ particle, as will be discussed in more detail in the next section.

In previous literature on the SLH model the $t_{\beta} \geq 1$ and $t_{\beta}<1$ cases are usually not distinguished, since a $t_{\beta} \rightarrow t_{\beta}^{-1}$ symmetry is tacitly assumed. Then only the $t_{\beta} \geq 1$ case is considered. However, strictly speaking this symmetry is only valid when the leptonic sector is not considered. Here we established clearly that if we consider the region favored by naturalness considerations, the $t_{\beta}<1$ case is disfavored by measurements of $m_{W}$ and $R$ observables. This is closely related to the breakdown of the $t_{\beta} \rightarrow t_{\beta}^{-1}$ symmetry in the lepton sector. Moreover, in previous literature [12,24], the sign combination of the rotation parameter $\delta_{D d}, \delta_{S s}$ was simply assumed to be (effectively) $\delta_{D d}^{-}, \delta_{S s}^{-}$, in order to suppress contributions to the electroweak precision observables. Here we also firmly establish this choice based on constraints from $R$ observables, combined with $m_{W}$ and naturalness considerations, keeping in mind that the constraint on $f$ has been pushed to around $7.5 \mathrm{TeV}$ due to updated LHC constraints. 


\section{PRODUCTION AND DECAY OF THE PSEUDOAXION}

With the preparations made in the previous three sections, we are now ready to calculate the production and decay of the pseudoaxion. We will restrict ourselves to the region $2 m_{t} \lesssim m_{\eta} \lesssim 1 \mathrm{TeV}$, which is favored by naturalness considerations. All of the related partial width formulas are given in Appendix B.

\section{A. Decay of the pseudoaxion}

For $\eta$ in the mass range $2 m_{t} \lesssim m_{\eta} \lesssim 1 \mathrm{TeV}$, it can always decay into $t \bar{t}, g g, \gamma \gamma$ channels. (The $W W, Z Z, Z \gamma$ channels are also possible and may have comparable branching ratios compared to $\gamma \gamma$. However, from a detection point of view, it is preferable to consider further decays into leptons in these channels, leading to an additional suppression by the leptonic branching. For simplicity we will not consider these channels further in this work.) $\eta \rightarrow \mathrm{ZH}$ is highly suppressed, since the antisymmetric $Z H \eta$ vertex is suppressed to $\mathcal{O}\left(\frac{v^{3}}{f^{3}}\right)$ while the symmetric $Z H \eta$ vertex does not contribute, as pointed out in Sec. III. If the new fermions $D, S, N$ are heavy enough such that they cannot appear as decay products of $\eta$, then we are left with only the $t \bar{t}, g g, \gamma \gamma$ channels. Nevertheless, we should keep in mind that when $f$ and $m_{\eta}$ are given, the partial widths of these channels still depend on the masses of the additional heavy quarks $T, D, S$ which do not appear as decay products of $\eta$. First, the $\eta \rightarrow t \bar{t}$ decay is controlled by the rotation parameter $\delta_{t}$, which in turn depends on the top partner mass. The loop-induced decays $\eta \rightarrow g g, \gamma \gamma$ have contributions from both the top quark and the heavy quark partners $T, D, S$. The top quark contribution again depends on $\delta_{t}$ while the $T, D, S$ contributions depend on the $\eta T \bar{T}, \eta D \bar{D}$, $\eta S \bar{S}$ couplings which are proportional to the corresponding rotation parameters times the quark partner mass. Experimentally, the current lower bound for the light-flavor quark partners $D$ and $S$ is around $700 \mathrm{GeV}$ [34]. Thus, for a heavy enough $\eta$ the $\eta \rightarrow D d$, Ss channels are still possible if the mass of $D$ or $S$ is close to the lower bound. To be definite, we will consider four benchmark scenarios.

(1) Case A: $f=8 \mathrm{TeV}, m_{T}=m_{D}=m_{S}=3 \mathrm{TeV}$, all $m_{N}>m_{\eta}$.

(2) Case B: $f=8 \mathrm{TeV}, m_{\eta}=500 \mathrm{GeV}, m_{D}=m_{S}=m_{T}$, all $m_{N}>m_{\eta}$.

(3) Case C: $f=8 \mathrm{TeV}, m_{T}=3 \mathrm{TeV}, m_{D}=700 \mathrm{GeV}$, $m_{S}=1 \mathrm{TeV}$, all $m_{N}=150 \mathrm{GeV}$.

(4) Case D: $f=8 \mathrm{TeV}, m_{\eta}=500 \mathrm{GeV}, m_{D}=m_{S}=m_{T}$, all $m_{N}=150 \mathrm{GeV}$.

For each case, there are two allowed sign combinations for the rotation parameters $\left(\delta_{t}, \delta_{D d}, \delta_{S s}\right):(+,-,-)$ and $(-,-,-)$. Other choices are excluded by electroweak precision measurements, if we are only interested in parameter regions favored by naturalness considerations. Therefore, in the following we will use Case $\mathrm{A}+$, Case A-, etc., to indicate the sign choice of $\delta_{t}$ in each case [see Eq. (52)].

The total width and branching ratios of $\eta$ are shown in Figs. 5 and 6 for Case A and Case B, respectively. In these two cases, the additional fermion partners $D, S, N$ are not light enough to appear as decay products of $\eta$, and therefore we are left with the standard $\eta \rightarrow t \bar{t}, g g, \gamma \gamma$ channels. From the figures it is clear that $\eta$ can be viewed as a narrow-width particle; however, the width is not small enough to give rise to displaced vertices. In both Case A and Case B and for both sign choices, $\eta$ decays almost $100 \%$ to $t \bar{t}$, with only very small branching ratios to $g g[\mathcal{O}(0.1 \%)]$ and $\gamma \gamma$ $[\mathcal{O}(0.001 \%)]$. Here (and in the following) all of the partial widths are calculated at LO, but it is obvious that the inclusion of higher-order radiative corrections has little effect on the whole picture. From a detection point of view, this situation is somewhat unfortunate since the dominant channel $t \bar{t}$ suffers from huge background at hadron colliders, while the clean channel $\gamma \gamma$ has an extremely small branching ratio. It is natural to ask how the situation will change if any of $D, S, N$ is light enough, such that exotic channels like $\eta \rightarrow N N, N \nu, D d$, Ss could be open. This is embodied in Cases C and D and we show the
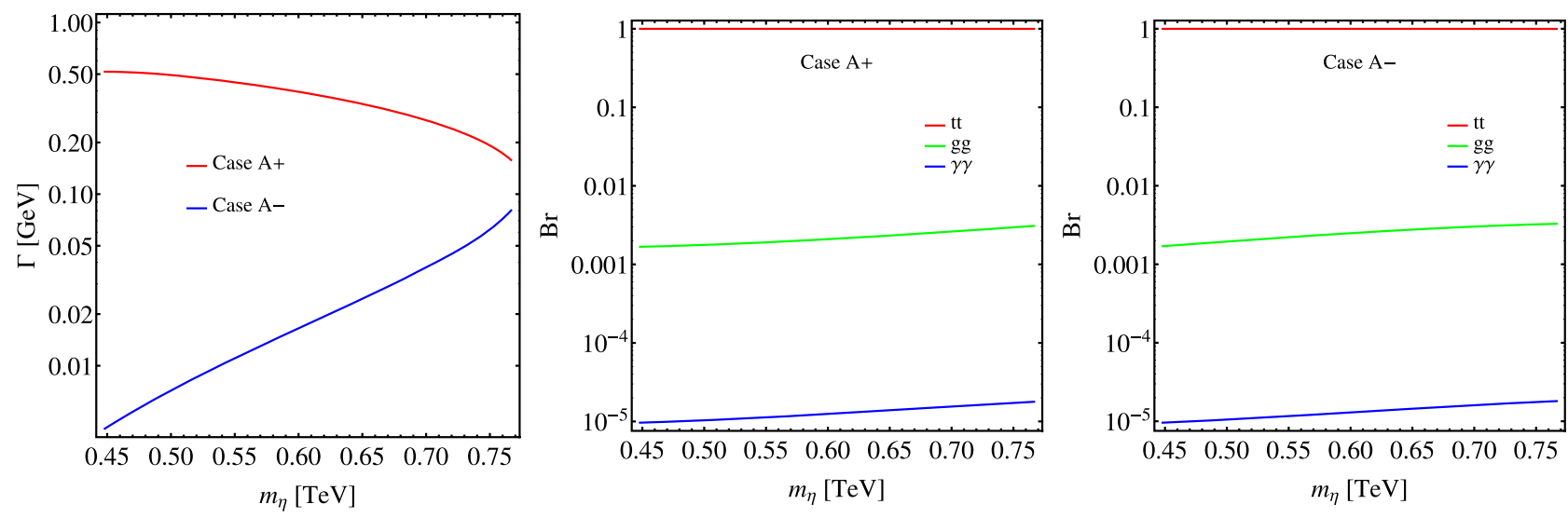

FIG. 5. Total width $\Gamma$ and decay branching ratios of $\eta$ in Case A. 

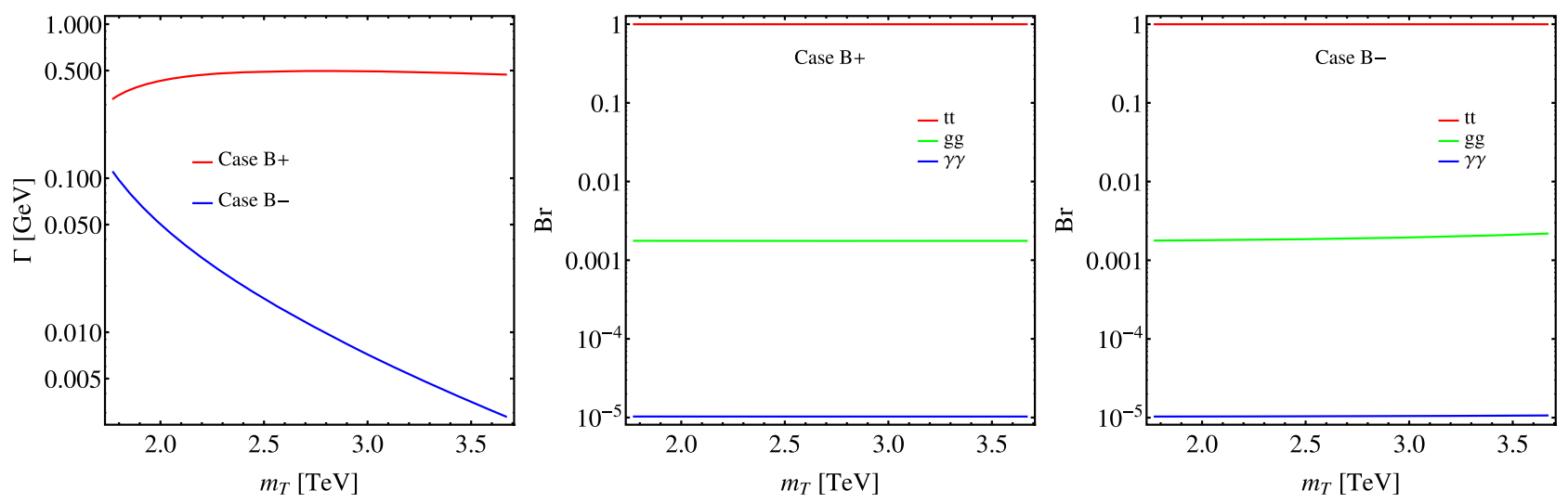

FIG. 6. Total width $\Gamma$ and decay branching ratios of $\eta$ in Case B.

corresponding branching ratio plots in Fig. 7. Nevertheless, the exotic channels contribute at most a few percent in terms of branching ratio, and therefore are of little use for $\eta$ detection even if any of $D, S, N$ is light enough. This can be understood from the interaction Lagrangian containing the $\eta D d, \eta S s$ and $\eta N \nu, \eta N N$ vertices. The $\eta D d$ vertex is shown in Eq. (62). When $\eta \rightarrow D d$ is open, $\frac{M_{D}}{v}$ is an $\mathcal{O}(1)$ quantity, and therefore from Eq. (62) we may recognize that the $\eta D d$ coupling can be considered as being relatively suppressed by $\mathcal{O}\left(\frac{v}{f}\right)$ compared to the $\eta t \bar{t}$ vertex. This leads to the suppression of the $\eta \rightarrow D d$ channel. The $\eta N \nu$ coupling is relatively suppressed by $\mathcal{O}\left(\frac{v}{f}\right)$ compared to the $\eta N N$ coupling, as can be seen from Eq. (60). However, when $\eta \rightarrow N N$ is open, $M_{N n}$ can be at most $\mathcal{O}(v)$. Moreover, the $\eta N N$ coupling suffers from a $t_{\beta}$ suppression. Therefore, numerically the $\eta \rightarrow N N$ channel is much more suppressed compared to the $\eta \rightarrow t \bar{t}$ channel.

\section{B. Decay of the top partner}

The pseudoaxion may appear as a decay product of some additional heavy particles in the model. Among the additional particles in the SLH only $Z^{\prime}$ and $T$ are closely related
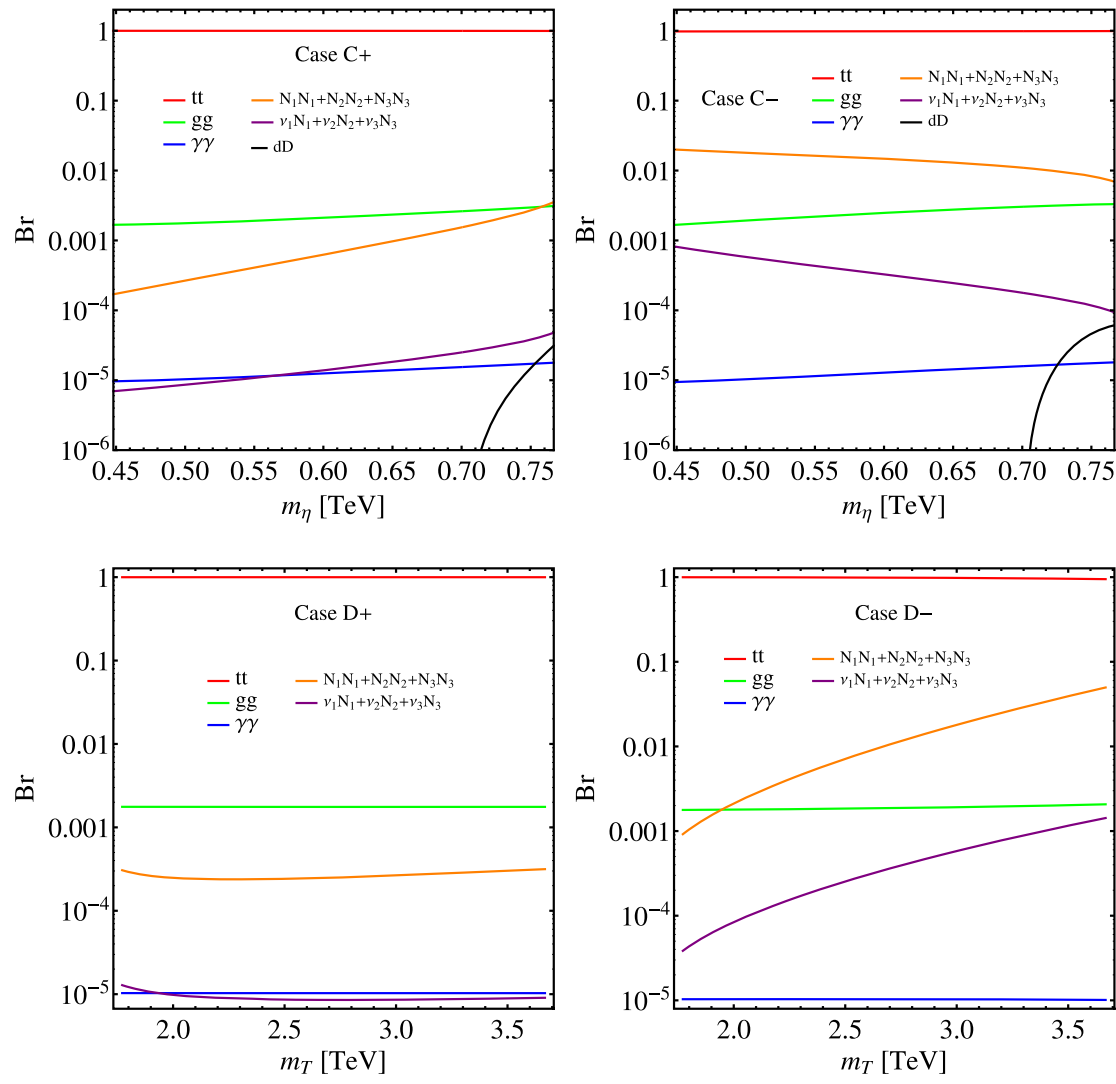

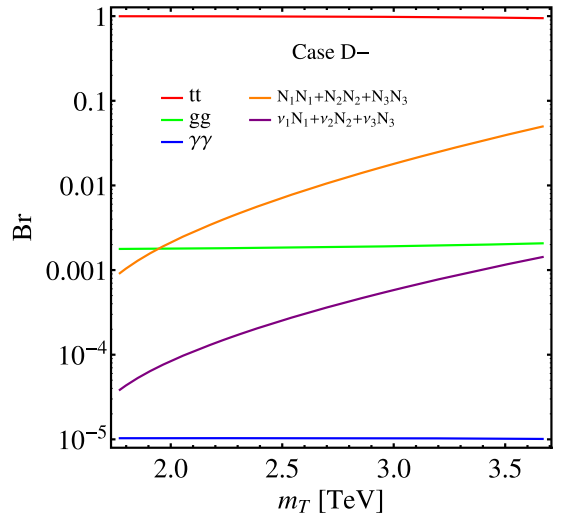

FIG. 7. Decay branching ratios of $\eta$ in Case C and Case D. 

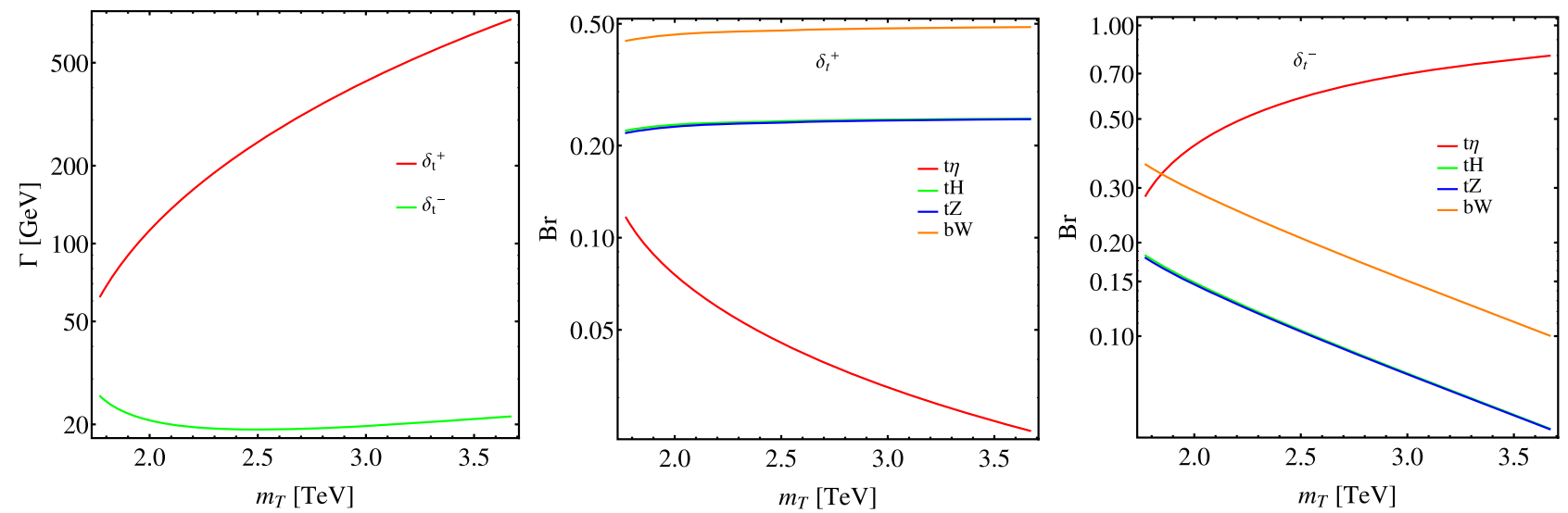

FIG. 8. Total width $\Gamma$ and decay branching ratios of $T$ in the SLH. We assume $f=8 \mathrm{TeV}$ and $m_{\eta}=500 \mathrm{GeV}$. Note that in the considered mass range $T \rightarrow b X, t Y$ channels are not open.

to EWSB and naturalness favors small $Z^{\prime}$ and $T$ masses within theoretical constraints. In this subsection we consider the decay of the top partner. The possibility of $T \rightarrow$ $t+a$ (where $T$ and $a$ denote the top partner and a pNGB in the context of composite Higgs models) has been investigated in the literature [35-37]. Here we focus on the situation in the SLH. To be specific, we fix $f=8 \mathrm{TeV}$ and $m_{\eta}=500 \mathrm{GeV}$ and then plot the total width and branching ratios of $T$ as functions of the top partner mass $m_{T}$ in Fig. 8.
Both $\delta_{t}^{+}$and $\delta_{t}^{-}$possibilities are considered. Note that when $m_{T}$ is also given, according to the mass relation $t_{\beta}$ can be calculated, which in turn determines the total width and branching ratios. The relation $\operatorname{Br}(T \rightarrow b W)=2 \operatorname{Br}(T \rightarrow$ $t H)=2 \mathrm{Br}(T \rightarrow t Z)$ holds to a good approximation. In the $\delta_{t}^{+}$case, $\operatorname{Br}(T \rightarrow t \eta$ ) is small (not larger than $10 \%$ for $m_{T}>2 \mathrm{TeV}$ ) and decreases with the increase of $m_{T}$. In the $\delta_{t}^{-}$case, $\operatorname{Br}(T \rightarrow t \eta)$ is sizable and becomes dominant (larger than 50\%) for $m_{T} \gtrsim 2.2 \mathrm{TeV}$. Another interesting
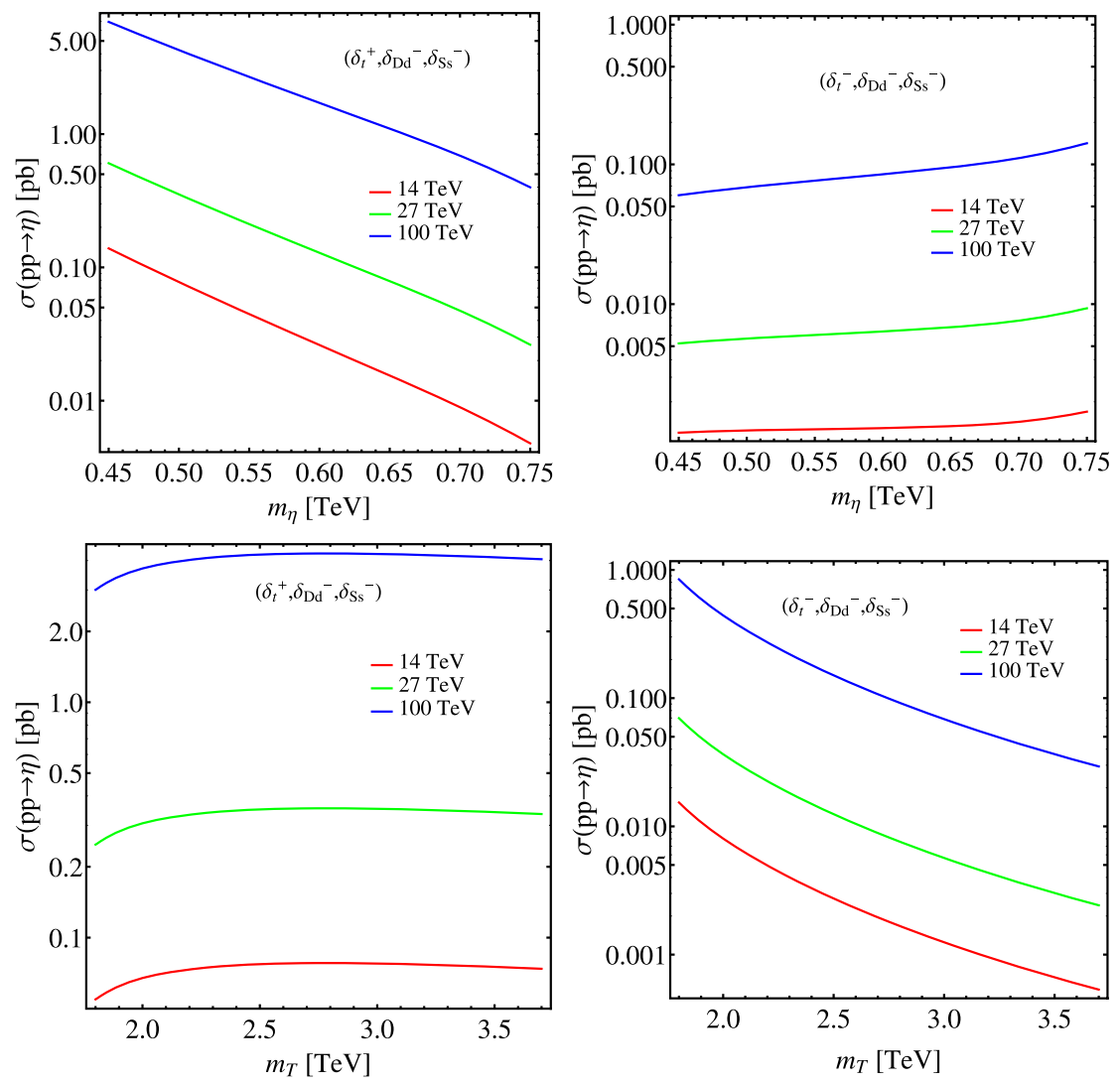

FIG. 9. Gluon-fusion production cross section of $\eta$ as a function of $m_{\eta}$ (upper panel, assuming $m_{T}=m_{D}=m_{S}=3 \mathrm{TeV}$ ) or $m_{T}$ (lower panel, assuming $m_{D}=m_{S}=m_{T}$ and $\left.m_{\eta}=500 \mathrm{GeV}\right)$. The sign combination of $\left(\delta_{t}, \delta_{D d}, \delta_{S s}\right)$ is indicated in each plot. 

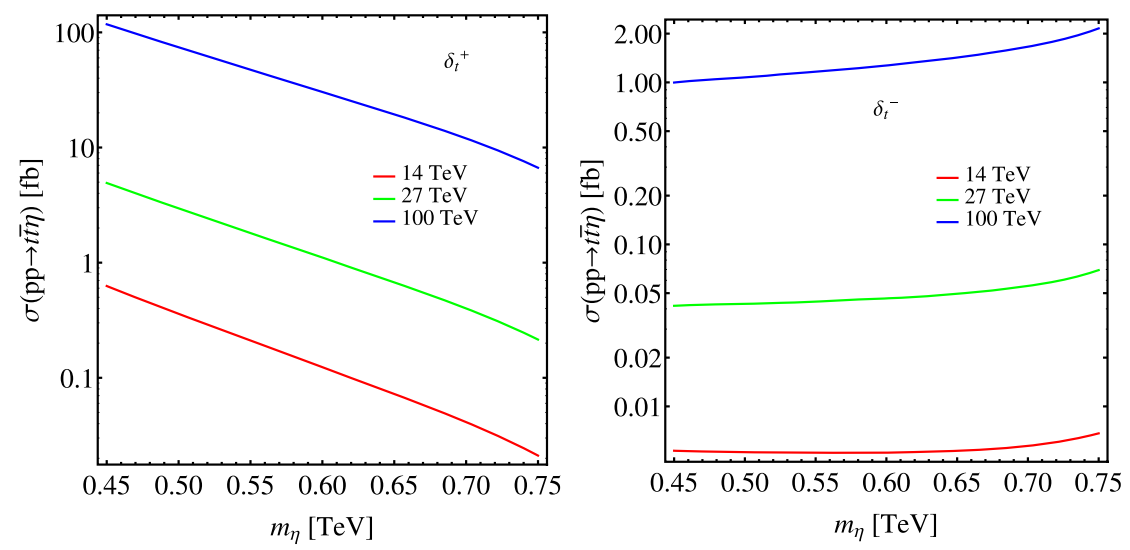

FIG. 10. Production cross section of $p p \rightarrow t \bar{t} \eta$ as a function of $m_{\eta}$. We assume $f=8 \mathrm{TeV}$ and $m_{T}=3 \mathrm{TeV}$.

and important feature concerns the total width of $T$. In the $\delta_{t}^{-}$case, the total width is around $20 \mathrm{GeV}$ which makes the narrow-width approximation valid to high precision. In the $\delta_{t}^{+}$case, the total width increases with $m_{T}$. For $m_{T} \approx$ $3.5 \mathrm{TeV}$ the total width increases to around $500 \mathrm{GeV}$. In this case $\Gamma / M \lesssim 20 \%$ and the narrow-width approximation still roughly holds, if the phase space is large enough. However, the width will have an appreciable impact on the invariant mass distribution of the $T$ decay products.

\section{Direct production of the pseudoaxion}

The pseudoaxion can be directly produced via the gluonfusion mechanism at hadron colliders. The particles running in the loop now contain $t, T, D, S$. In the calculation of the production cross section, ${ }^{10}$ we consider the $14 \mathrm{TeV}$ (HL-)LHC, the $27 \mathrm{TeV}$ HE-LHC, and the $100 \mathrm{TeV}$ FCChh. The production cross sections are plotted in Fig. 9 as functions of $m_{\eta}$ or $m_{T}$, with other parameters described in the figure caption. Although the production cross section may reach $\mathcal{O}(\mathrm{pb})$ in certain regions of parameter space, unfortunately when combined with $\eta$ decay it becomes very difficult to detect in the gluon-fusion channel. The dominant $t \bar{t}$ decay mode suffers from huge background, while the $\gamma \gamma$ decay mode has only a $\mathcal{O}\left(10^{-5}\right)$ branching ratio.

Another way to directly produce $\eta$ is through the $p p \rightarrow t \bar{t} \eta$ channel. We plot the production cross section as a function of $m_{\eta}$ in Fig. 10, for three center-of-mass energies and both $\delta_{t}^{+}$and $\delta_{t}^{-}$. Here we fix $f=8 \mathrm{TeV}$ and $m_{T}=3 \mathrm{TeV}$, and therefore for a given $m_{\eta}, t_{\beta}$ (and $\delta_{t}^{ \pm}$) is also determined. The cross section in the $\delta_{t}^{-}$case is much

\footnotetext{
${ }^{10}$ For simplicity, in this work all of the cross sections are calculated at LO using MADGRAPH5_AMC@NLO [38] and FEYNRules [39]. We use the MSTW2008lo68cl parton distribution function [40]. For $2 \rightarrow 1$ production, the renormalization and factorization scale is taken to be the rest mass of the $s$-channel resonance. Otherwise, the renormalization and factorization scale is taken to be the sum of the transverse masses of final-state particles (before resonance decay) divided by 2 .
}

smaller than that in the $\delta_{t}^{+}$case. Even in the $\delta_{t}^{+}$case the detection of the $p p \rightarrow t \bar{t} \eta$ process is still very difficult. For instance, if we take $m_{\eta}=450 \mathrm{GeV}$, then in the $\delta_{t}^{+}$case the cross section reaches only about $0.6 \mathrm{fb}$ at $14 \mathrm{TeV}$ and $100 \mathrm{fb}$ at $100 \mathrm{TeV}$. When we consider $\eta \rightarrow t \bar{t}$ decay, there exists the SM four-top production as an irreducible background, with a cross section of about $10 \mathrm{fb}$ at $14 \mathrm{TeV}$ and $5000 \mathrm{fb}$ at $100 \mathrm{TeV}$. Unfortunately, since $m_{\eta}$ is not far above the $2 m_{t}$ threshold, we do not expect large differences in kinematical features between the $p p \rightarrow t \bar{t} \eta$ signal and the SM four-top background, making the discrimination very difficult. With larger $m_{\eta}$ (say, $1 \mathrm{TeV}$ ), the top pair from $\eta$ decay can be boosted, with an invariant mass distribution that peaks around a high value, which can facilitate the discrimination from SM backgrounds. However, the cross section for such a heavy $\eta$ becomes very small. Therefore, we do not expect $p p \rightarrow t \bar{t} \eta$ to be a promising channel for future $\eta$ detection in the SLH.

\section{Pseudoaxion production from top partner decay}

The above discussion shows that it is very difficult to detect $\eta$ via the gluon-fusion and $t \bar{t} \eta$-associated production channels. It is therefore natural to consider alternative $\eta$ production mechanisms, such as decay from heavier particles. In the SLH, particles that can be heavier than $\eta$ are $T, D, S, N, Z^{\prime}, X$ and $Y$. Here we will concentrate on $T$, which is most tightly connected to EWSB. We will briefly comment on the possibility of detecting $\eta$ from other heavy particle decays in the next subsection.

Under current constraints, the lower bound on $m_{T}$ is already larger than the largest possible value of $m_{\eta}$ plus $m_{t}$, and therefore the exotic decay channel $T \rightarrow t \eta$ will always be open. The branching fraction of $T \rightarrow t \eta$ has been discussed (see Fig. 8). Here we focus on top partner production. Two major production mechanisms are pair production through QCD interaction, and single production through the $T b W$ vertex. Pair production has the virtue of being model independent, while single production depends on the value of $\delta_{t}$. In Fig. 11 we present the cross sections of 

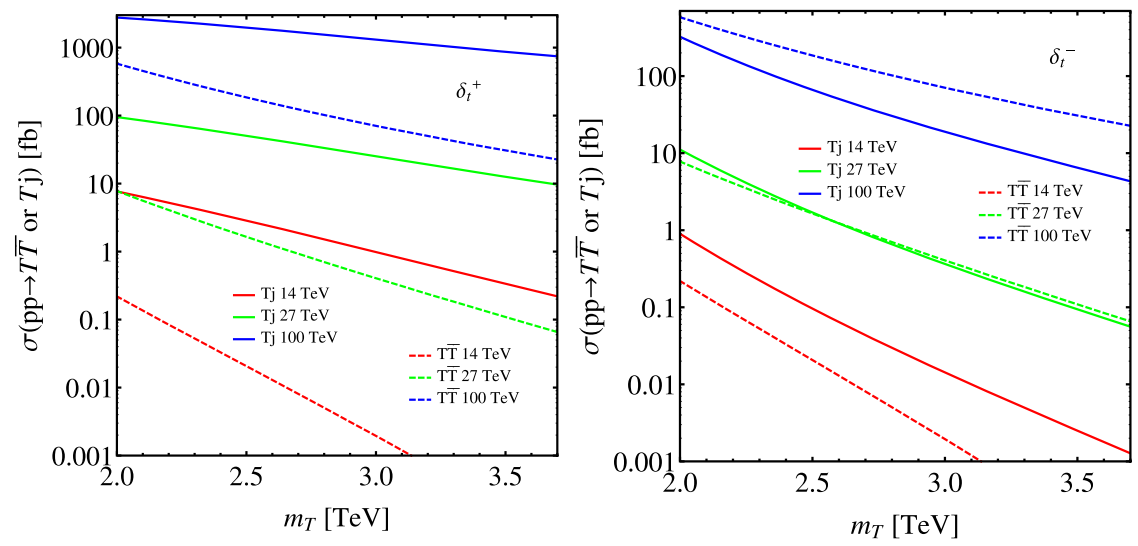

FIG. 11. Production cross sections of $p p \rightarrow T \bar{T}$ and $p p \rightarrow T j$ as functions of $m_{T}$. We assume $f=8 \mathrm{TeV}$ and $m_{\eta}=500 \mathrm{GeV}$. For $p p \rightarrow T j$, the contribution from $p p \rightarrow \bar{T} j$ is also included.

$p p \rightarrow T \bar{T}$ and $p p \rightarrow T j+\bar{T} j$ for both $\delta_{t}^{+}$and $\delta_{t}^{-}$, as functions of $m_{T}$ while we fix $f=8 \mathrm{TeV}, m_{\eta}=500 \mathrm{GeV}$. Three center-of-mass energies $(14,27,100 \mathrm{TeV})$ are considered. Whether pair or single production delivers a larger cross section depends on the sign choice for $\delta_{t}$ and the center-of-mass energy. In the $\delta_{t}^{+}$case, for all three center-of-mass energies the single production cross section is larger. In the $\delta_{t}^{-}$case, at $14 \mathrm{TeV}$ single production is larger since pair production is highly suppressed by phase space. At $27 \mathrm{TeV}$ pair production and single production become comparable, while for $100 \mathrm{TeV}$ collider energy pair production dominates.

To detect $\eta$ we would also like to consider the top partner decay $T \rightarrow t \eta$ that follows the pair or single production of $T$. The associated cross sections are plotted as functions of $m_{T}$ in Fig. 12, using the narrow-width approximation, for
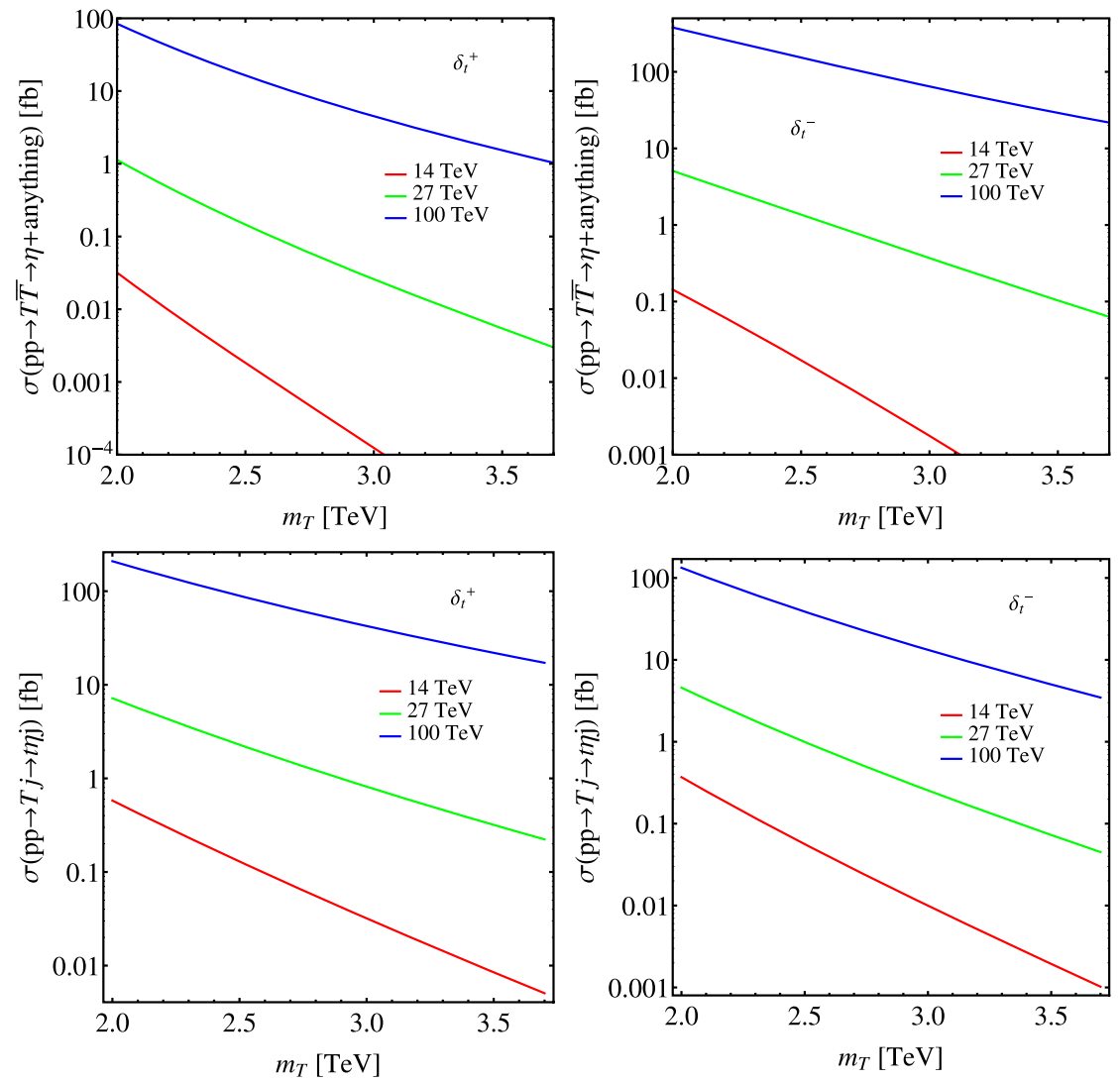

FIG. 12. Cross sections of $p p \rightarrow T \bar{T} \rightarrow \eta+$ anything and $p p \rightarrow T j \rightarrow \eta+$ anything as functions of $m_{T}$. We assume $f=8 \mathrm{TeV}$ and $m_{\eta}=500 \mathrm{GeV}$. For $p p \rightarrow T j$, the contribution from $p p \rightarrow \bar{T} j$ is also included. 
both $\delta_{t}^{+}$and $\delta_{t}^{-}$. For definiteness we take $f=8 \mathrm{TeV}$, $m_{\eta}=500 \mathrm{GeV}$. To be precise, the plotted cross sections are defined by (for $p p \rightarrow T j$, the contribution from $p p \rightarrow$ $\bar{T} j$ is also included)

$$
\sigma(p p \rightarrow T j \rightarrow \eta+\text { anything })=\sigma(p p \rightarrow T j) \times \operatorname{Br}(T \rightarrow t \eta),
$$

$$
\begin{aligned}
\sigma(p p & \rightarrow T \bar{T} \rightarrow \eta+\text { anything }) \\
= & 2 \sigma(p p \rightarrow T \bar{T}) \times \operatorname{Br}(T \rightarrow t \eta)(1-\operatorname{Br}(T \rightarrow t \eta)) \\
& +\sigma(p p \rightarrow T \bar{T}) \times \mathrm{Br}^{2}(T \rightarrow t \eta) .
\end{aligned}
$$

For the purpose of $\eta$ detection, let us consider using the $\eta \rightarrow t \bar{t}$ channel, which has an almost $100 \%$ branching fraction. Then, the $\eta$ production from top partner decays generically leads to a multitop $(\geq 3)$ signature. Moreover, the top quarks will be boosted since $m_{T} \gg m_{t}+m_{\eta}$. For example, suppose a $2 \mathrm{TeV}$ top partner is produced with little boost in the lab frame and then decays into $t+\eta$. At this step, $t$ and $\eta$ roughly share the rest energy of the top partner and therefore will each have about $1 \mathrm{TeV}$ energy. The $\eta$ boson then further decays into $t$ and $\bar{t}$, each of which roughly has an energy of about $0.5 \mathrm{TeV}$. All three top quarks are boosted: the first one will have a decay $(t \rightarrow b W)$ cone size approximated by $\sim 2 m_{t} / E_{t} \simeq 0.4$, while the second and third top have $\sim 2 m_{t} / E_{t} \simeq 0$.8. Furthermore, the second and third top quark decaying from $\eta$ are close to each other, separated by approximated $\sim 2 m_{\eta} / E_{\eta} \simeq 0.8$.

In the single production case, the signature will be $3 t+j$, in which the first top is highly boosted while the second and third are still somewhat boosted and close to each other. One can make use of such kinematics to discriminate from QCD backgrounds. The most serious background is perhaps multitop production. One may be able to reduce the background using the boosted techniques [41]. In the pair production case, if we consider one top partner decaying into $t \eta$ with the other decaying into $b W$, we obtain a signature of $3 t+b+W$ in which the top quarks and also the $W$ boson will be boosted. In both the single and pair production channels, the invariant mass peaks at $m_{T}$ and $m_{\eta}$ will also be helpful in discriminating between the signal and background. Nevertheless, a full signal-background analysis using boosted-top techniques is beyond the scope of the present work.

From Fig. 12, we see that the cross sections at the $14 \mathrm{TeV}$ (HL-)LHC for all of these channels are very small $(<1 \mathrm{fb})$, making the detection very difficult. Nevertheless, with the increase of collider energy, the signal cross sections increase significantly. For example, at the $100 \mathrm{TeV}$ FCC-hh, for both $\delta_{t}^{+}$and $\delta_{t}^{-}$and pair and single production channels, at relatively small $m_{T}$ the cross sections could reach $\mathcal{O}(100 \mathrm{fb})$. In the $\delta_{t}^{+}$case, the single production (with the top partner decaying to $t \eta$ ) provides a cross section of about $200 \mathrm{fb}$, which is larger than the pair production channel. In the $\delta_{t}^{-}$case, the pair production (with one top partner decaying to $t \eta$ ) provides a cross section of about $400 \mathrm{fb}$, which is however larger than the single production channel.

In principle, top partner production and decay provide a way to measure $t_{\beta}$ (which is important for testing the SLH mass relation) and also discriminate between the $\delta_{t}^{+}$and $\delta_{t}^{-}$ cases. In practice, we may consider the partial-width ratio $R_{\eta} \equiv \frac{\Gamma(T \rightarrow t \eta)}{\Gamma(T \rightarrow b W)}$ as both an indicator of the sign choice for $\delta_{t}$ and a way to measure $\delta_{t}$, which in turn determines $t_{\beta}$. $\delta_{t}$ can also be determined from $p p \rightarrow T j$ production since the cross section is proportional to $\delta_{t}^{2}$. Furthermore, in the $\delta_{t}^{+}$ case the total width of $T$ could reach $\mathcal{O}(100 \mathrm{GeV})$, which may have an impact on the invariant mass distribution of $T$ decay products (e.g., $b W$ ). Measurement of the $T$ total width in principle could also help determine the value of $\delta_{t}$. However, even if $\delta_{t}$ is determined (including the sign choice), the determination of $t_{\beta}$ and the test of the mass relation still require the measurement of $f$ and $m_{\eta}$, which can be obtained if we are able to measure the masses of $Z^{\prime}$ and $\eta$ particles.

\section{E. Comments on other channels}

Currently the SLH is stringently constrained by the LHC $Z^{\prime} \rightarrow l l$ search; nevertheless, it also means that if the SLH was realized in nature, the $Z^{\prime} \rightarrow l l$ signature would be the first place that we might expect the appearance of new physics. Then it would also be important to consider whether we may detect $\eta$ as a decay product of $Z^{\prime}$. Two channels might be considered: $Z^{\prime} \rightarrow \eta H$ and $Z^{\prime} \rightarrow \eta Y$. However, it turns out they give too small branching fractions: $\operatorname{Br}\left(Z^{\prime} \rightarrow \eta H\right)<0.01$ and $\operatorname{Br}\left(Z^{\prime} \rightarrow \eta Y\right)<10^{-4}$. This is regardless of whether the $Z^{\prime} \rightarrow D D, S S, N N$ channels are kinematically allowed. Therefore, it is not preferable to consider detecting $\eta$ from $Z^{\prime}$ decay.

If kinematically allowed, we might also consider $D \rightarrow d \eta, S \rightarrow s \eta, N \rightarrow \nu \eta$ decays. However, these decay channels also suffer from small branching fractions, since the $\eta D d, \eta S s, \eta N \nu$ couplings are $\mathcal{O}\left(\frac{v}{f}\right)$ suppressed compared to $H D d, H S s, H N \nu$ couplings [see Eqs. (60) and (62)]. For example, $D$ will dominantly decay to $u W, d Z$, $d H$, with only $\operatorname{Br}(D \rightarrow d \eta)<1 \%$, for the benchmark point $f=8 \mathrm{TeV}, m_{T}=2 \mathrm{TeV}, m_{\eta}=0.5 \mathrm{TeV}$ and any value of $m_{D}$. Here $\delta_{D d}^{-}$is assumed, to be consistent with electroweak precision constraints. As for $D$ production, for the $\delta_{D d}^{-}$case, there is a $t_{\beta}^{-1}$ suppression for single $D$ production, and therefore $D$ pair production is more promising. Moreover, current collider constraints on the $D$ mass are not stringent, such that $m_{D}=700 \mathrm{GeV}$ is still allowed [34]. Therefore, if $m_{D}$ is as light as $700 \mathrm{GeV}$, the large $p p \rightarrow D \bar{D}$ production cross section could compensate for the small $D \rightarrow d \eta$ branching fraction, leading to a sizable $\eta$ production rate. 

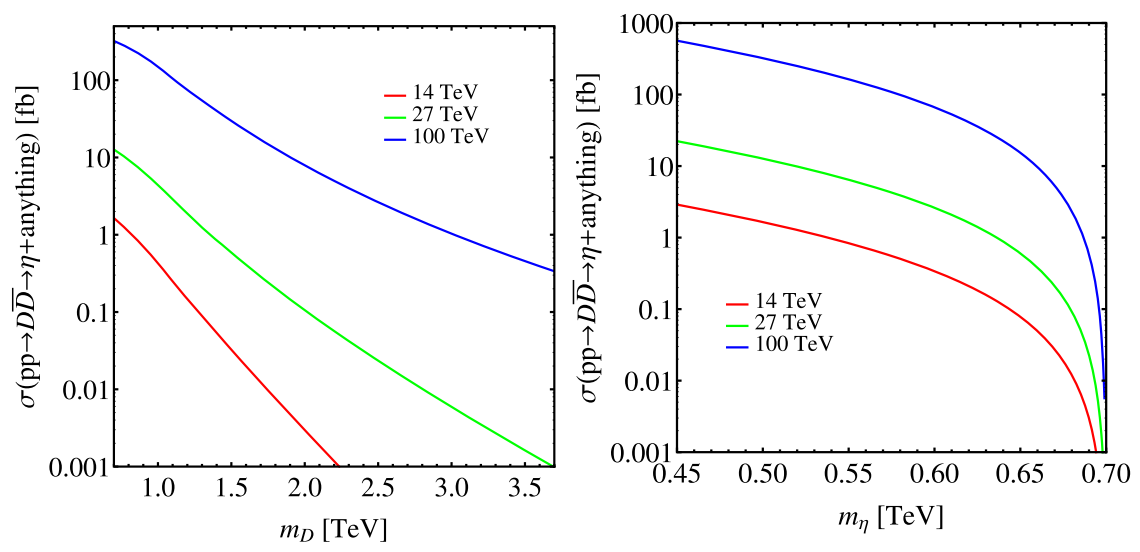

FIG. 13. Production cross section of $p p \rightarrow D \bar{D} \rightarrow \eta+$ anything as a function of $m_{D}$ (left) and $m_{\eta}$ (right). For the left plot, we assume $f=8 \mathrm{TeV}, m_{T}=3 \mathrm{TeV}, m_{\eta}=500 \mathrm{GeV}$. For the right plot, we assume $f=8 \mathrm{TeV}, m_{T}=3 \mathrm{TeV}, m_{D}=700 \mathrm{GeV}$.

At the $100 \mathrm{TeV}$ FCC-hh, the $\eta$ production cross section from $D$ decay, $\sigma(p p \rightarrow D \bar{D} \rightarrow \eta+$ anything) could also reach more than $100 \mathrm{fb}$ for $m_{D}$ not much larger than $700 \mathrm{GeV}$ (see Fig. 13). This is comparable to the $\eta$ cross section from top partner production, and in principle could also be used to measure $t_{\beta}$. The expected signature would be $t \bar{t}+2 j+W / Z / H$, in which the $W / Z / H$ should be boosted. The existence of various intermediate resonances would be helpful in discriminating signal and background. Nevertheless, we should be aware that naturalness does not offer any guidance on the preferred value of $m_{D}$. This is different from the case of $m_{T}$, in which naturalness clearly favors a lighter top partner. The case of $p p \rightarrow S \bar{S}$ production with $S \rightarrow s \eta$ decay is completely similar to the above discussion of $D$ production and decay. For $N$, $\operatorname{Br}(N \rightarrow \nu \eta)$ is also very small (less than $1 \%$ for the benchmark point $f=8 \mathrm{TeV}, m_{T}=2 \mathrm{TeV}, m_{\eta}=0.5 \mathrm{TeV}$ and any value of $m_{N}$ ). Moreover, $N$ does not have QCD pair production channels like $D, S$, and therefore it is difficult to detect $\eta$ from $N$ decay at hadron colliders.

The $X, Y$ gauge bosons in the SLH may have decays like $X \rightarrow \eta W$ and $Y \rightarrow H \eta$. However, the single production cross sections of $X, Y$ at hadron colliders are highly suppressed, and we need to rely on production with other heavy particles (heavy gauge bosons or quark partners) [24]. Since $X, Y$ bosons are quite heavy (with masses of about $0.8 m_{Z^{\prime}}$ ), their production with other heavy particles would be limited by phase space while their decays are expected to be dominated by fermionic final states. Therefore, we do not consider $\eta$ production from $X, Y$ decays as promising channels for $\eta$ detection.

\section{DISCUSSION AND CONCLUSIONS}

The simplest little Higgs model provides a simple way to concretely realize the collective symmetry breaking mechanism, in order to alleviate the Higgs mass naturalness problem. In the scalar sector, its particle content is very economical, since besides the $C P$-even Higgs which should serve as the $125 \mathrm{GeV}$ Higgs-like particle, the only additional scalar particle is the pseudo-Nambu-Goldstone particle $\eta$ associated with a remnant global $U(1)$ symmetry. The detection of $\eta$ is important since its mass enters into the crucial SLH mass relation and it will also play an important role in discriminating SLH from other new physics scenarios. In this work we have been concerned with the production and decay of the $\eta$ particle at future hadron colliders. We found that for natural regions of parameter space, $m_{\eta}$ is larger than $2 m_{t}$ and decays almost exclusively to $t \bar{t}$, and $\operatorname{Br}(\eta \rightarrow \gamma \gamma)$ is too small to be considered promising for detection. Also, it is very difficult to detect $\eta$ in the direct production channels $p p \rightarrow \eta$ (gluon fusion) and $p p \rightarrow t \bar{t} \eta$. Channels that are worth further consideration include $\eta$ production from heavy quark partner $(T, D, S)$ decays, in which the heavy quark partner might be singly (for $T$ ) or pair produced. The corresponding $\eta$ production cross section at the $100 \mathrm{TeV}$ FCC-hh could reach $\mathcal{O}(100 \mathrm{fb})$ for certain ranges of parameter space that are allowed by current constraints, while at the $14 \mathrm{TeV}$ (HL-)LHC the rate might be too small for detection. However, the detection prospects in these channels (at $100 \mathrm{TeV}$ ) might still be challenging since the final states are quite complicated, including multitop-associated production with other objects, in which one or more of them could be boosted, requiring sophisticated tagging techniques. At the same time, the SM background also enjoys a large increase with the collider energy, with more a complicated hadronic environment. The aim of this paper was to examine the $\eta$ production channels with a LO estimate of the $\eta$ cross sections in the relatively promising ones as functions of the model parameters, keeping in mind the most up-to-date theoretical and experimental constraints (see Table II for a summary). We did not attempt to give a quantitative assessment of the collider sensitivities in these channels.

The phenomenology of the $\eta$ particle in the SLH was studied a long time ago in several papers (e.g., Refs. $[17,18,20,21])$. Compared to all of the previous 
TABLE II. Summary of $\eta$ production from $T, D(S)$ decays at the $100 \mathrm{TeV}$ FCC-hh. For $p p \rightarrow T j$, the contribution from $p p \rightarrow \bar{T} j$ is also taken into account. For $T \bar{T}, T j$ channels, the benchmark point is $f=8 \mathrm{TeV}, m_{T}=2 \mathrm{TeV}, m_{\eta}=500 \mathrm{GeV}$ while for $D \bar{D}$ channels, the benchmark point is $f=8 \mathrm{TeV}, m_{T}=3 \mathrm{TeV}, m_{\eta}=500 \mathrm{GeV}, m_{D}=700 \mathrm{GeV}$. When listing the signatures for $T \bar{T}, D \bar{D}$ channels we do not consider the situation in which both quark partners decay into $\eta+t$ or $\eta+j$, but this possibility is taken into account in the cross section values and plots.

\begin{tabular}{lcc}
\hline \hline Channel & Cross section at the benchmark point $(\sqrt{s}=100 \mathrm{TeV})(\mathrm{fb})$ & Signature \\
\hline$p p \rightarrow T \bar{T} \rightarrow \eta+$ anything & $84\left(\delta_{t}^{+}\right), 379\left(\delta_{t}^{-}\right)$ & $3 t+W+b$ or $4 t+Z / H$ \\
$p p \rightarrow T j \rightarrow \eta+$ anything & $209\left(\delta_{t}^{+}\right), 133\left(\delta_{t}^{-}\right)$ & $3 t+j$ \\
$p p \rightarrow D \bar{D} \rightarrow \eta+$ anything & 322 & $2 t+W / Z / H+2 j$ \\
\hline \hline
\end{tabular}

studies, the present paper is different in a few crucial aspects.

(1) Instead of working with the ad hoc assumption of no direct contribution to the scalar potential from the physics at the cutoff, we took into account in all calculations the crucial SLH mass relation (29) which is a reliable prediction of the SLH. Therefore, our prediction preserves all of the correlations required by theoretical consistency but does not depend on the choice of any fixed cutoff value such as $4 \pi f$.

(2) We have focused our attention on the parameter region favored by naturalness considerations. This region is characterized by small $m_{T}$ and large $t_{\beta}$ or $t_{\beta}^{-1}$. The favored $\eta$ mass is larger than $2 m_{t}$.

(3) We have taken into account the recent collider constraint on $f(f \gtrsim 7.5 \mathrm{TeV})$ which is much more stringent than the constraints obtained a long time ago. We also took into account the constraint from perturbative unitarity which sets an upper bound on the allowed value of $t_{\beta}$ or $t_{\beta}^{-1}$. These two factors determine the current lower bound on $m_{T}$ and crucially affect the largest cross section that can be achieved in all channels.

(4) Our study is based on an appropriate treatment of the diagonalization of the vector-scalar system in the SLH, and especially the field redefinition related to $\eta$. This affects the derivation of $\mathrm{ZH} \eta$ vertices and also $\eta$ coupling to fermions, which were not treated properly in previous works until Ref. [22].

(5) We also clarified the role played by the symmetric VSS vertices that appear in the Lagrangian and how they are compatible with the general principles, like field redefinition invariance and gauge independence.

From our study it turns out that the detection of $\eta$ at the $14 \mathrm{TeV}$ (HL-)LHC will be very difficult, and therefore a $p p$ collider with higher energy and larger luminosity, such as the $27 \mathrm{TeV}$ HE-LHC or even the $100 \mathrm{TeV}$ FCC-hh or SppC, is motivated to capture the trace of such an elusive particle. Moreover, generally we would expect some other SLH signatures (e.g., $Z^{\prime} \rightarrow l l, T \rightarrow b W$ or $D \rightarrow u W$ ) to show up earlier than $\eta$ signatures since $\eta$ signatures are usually very complicated (with multiple top quarks) and suffer from small rates. It is nonetheless important to study $\eta$ properties since they are crucial in testing the SLH mass relation and also provide a basis for model discrimination.

\section{ACKNOWLEDGMENTS}

We thank Yue-Lin Sming Tsai for helpful discussion. P. Y. T. was supported by the World Premier International Research Center Initiative (WPI), MEXT, Japan. This work was supported in part by the Natural Science Foundation of China (Grants No. 11635001 and No. 11875072), the China Postdoctoral Science Foundation (Grant No. 2017M610992), and the MoST of Taiwan under grant No. 105-2112-M-007-028-MY3 and No. 107-2112-M007-029-MY3.

\section{APPENDIX A: CONVENTION CONVERSION}

References [23,24] contain detailed treatments of the anomaly-free SLH model. However, they used different conventions and it is useful to establish a conversion rule to relate formulas between the two conventions. Reference [23] used the following covariant derivative expression:

$$
D_{\mu}=\partial_{\mu}-i g A_{\mu}^{a} T^{a}+i g_{x} Q_{x} B_{\mu}^{x}, \quad g_{x}=\frac{g t_{W}}{\sqrt{1-t_{W}^{2} / 3}}
$$

On the other hand, Ref. [24] used

$$
D_{\mu}=\partial_{\mu}+i g A_{\mu}^{a} T^{a}+i g_{x} Q_{x} B_{\mu}^{x}, \quad g_{x}=\frac{g t_{W}}{\sqrt{1-t_{W}^{2} / 3}} .
$$

Therefore, to convert between the two conventions, we need

$$
g \leftrightarrow-g, \quad t_{W} \leftrightarrow-t_{W}
$$

if we assume $g_{x} \leftrightarrow g_{x}$ and $A_{\mu}^{a} \leftrightarrow A_{\mu}^{a}, B_{\mu}^{x} \leftrightarrow B_{\mu}^{x}, T^{a} \leftrightarrow T^{a}$, $Q_{x} \leftrightarrow Q_{x}$. The transformations of $s_{W}$ and $c_{W}$ are still not determined. For convenience, we would like to identify the 
first-order gauge boson mass eigenstates $Z, Z^{\prime}, A$ in both conventions, namely,

$$
Z \leftrightarrow Z, \quad Z^{\prime} \leftrightarrow Z^{\prime}, \quad A \leftrightarrow A .
$$

Then, by comparing the first-order gauge boson mixing formulas in the two papers we are led to the following conversion rule for $s_{W}$ and $c_{W}$ :

$$
c_{W} \leftrightarrow c_{W}, \quad s_{W} \leftrightarrow-s_{W} .
$$

Using these rules, it is straightforward to convert between the two conventions. (Our present work adopts the same convention as in Ref. [23].) Then, e.g., the Lagrangian coefficient of $Z^{\prime} f \bar{f}$ couplings will acquire a minus sign during conversion since $g \leftrightarrow-g$. However, the expression for $\delta_{Z}$ [see Eq. (93)] remains the same since $c_{W} \leftrightarrow c_{W}$.

\section{APPENDIX B: PARTIAL-WIDTH FORMULAS}

Let us define

$$
F(x, y) \equiv \sqrt{(1+x+y)(1-x-y)(1+x-y)(1-x+y)} .
$$

In particular, we have

$$
F(0, x)=1-x^{2}, \quad \text { for }|x| \leq 1 .
$$

The partial-width formulas related to $\eta, T, D, S, N, Z^{\prime}$ decays are listed as follows.

(1) $\eta$ decay: Tree-level decay channels (to fermion final states):

$$
\begin{gathered}
\Gamma_{\eta \rightarrow t \bar{t}}=\frac{3 m_{\eta}}{8 \pi}\left(\frac{m_{t} \delta_{t}}{v}\right)^{2} \sqrt{1-\frac{4 m_{t}^{2}}{m_{\eta}^{2}}}, \\
\Gamma_{\eta \rightarrow d D}=\frac{3 m_{\eta}}{8 \pi}\left(\frac{m_{D}}{v}\right)^{2}\left(\delta_{D d}^{2}+\frac{v^{2}}{2 f^{2}}\right)^{2}\left(1-\frac{m_{D}^{2}}{m_{\eta}^{2}}\right)^{2}, \\
\Gamma_{\eta \rightarrow N \bar{N}}=\frac{m_{\eta} m_{N}^{2}}{16 \pi f^{2} t_{\beta}^{2}} \sqrt{1-\frac{4 m_{N}^{2}}{m_{\eta}^{2}}} \\
\Gamma_{\eta \rightarrow \nu N}=\frac{m_{\eta}}{8 \pi}\left(\frac{v m_{N}}{2 f^{2} s_{\beta}^{2}}\right)^{2}\left(1-\frac{m_{N}^{2}}{m_{\eta}^{2}}\right)^{2} .
\end{gathered}
$$

Here we adopt the notation $\Gamma_{\eta \rightarrow d D} \equiv \Gamma_{\eta \rightarrow d \bar{D}}+\Gamma_{\eta \rightarrow D \bar{d}}$ and $\Gamma_{\eta \rightarrow \nu N} \equiv \Gamma_{\eta \rightarrow \nu \bar{N}}+\Gamma_{\eta \rightarrow \bar{\nu} N}$.

Loop-induced decay channels:

$$
\begin{gathered}
\Gamma_{\eta \rightarrow g g}=\frac{m_{\eta}^{3} \alpha_{s}^{2}}{128 \pi^{3} v^{2}}\left|-\delta_{t} A_{\frac{1}{2}}\left(\tau_{t}\right)+\delta_{t} A_{\frac{1}{2}}\left(\tau_{T}\right)+\delta_{D d} A_{\frac{1}{2}}\left(\tau_{D}\right)+\delta_{S s} A_{\frac{1}{2}}\left(\tau_{S}\right)\right|^{2}, \\
\Gamma_{\eta \rightarrow \gamma \gamma}=\frac{m_{\eta}^{3} \alpha_{e m}^{2}}{2304 \pi^{3} v^{2}}\left|-4 \delta_{t} A_{\frac{1}{2}}\left(\tau_{t}\right)+4 \delta_{t} A_{\frac{1}{2}}\left(\tau_{T}\right)+\delta_{D d} A_{\frac{1}{2}}\left(\tau_{D}\right)+\delta_{S S} A_{\frac{1}{2}}\left(\tau_{S}\right)\right|^{2} .
\end{gathered}
$$

Here $\tau_{f} \equiv m_{\eta}^{2} / 4 m_{f}^{2}$ and for $f=T, D, S$ we have $\tau_{f} \ll 1$. The function $A_{\frac{1}{2}}(\tau) \equiv 2 f(\tau) / \tau$, where

$$
f(\tau)= \begin{cases}\arcsin ^{2} \sqrt{\tau} & (\tau \leq 1), \\ -\frac{1}{4}\left(\ln \frac{1+\sqrt{1-1 / \tau}}{1-\sqrt{1-1 / \tau}}-\mathrm{i} \pi\right)^{2} & (\tau>1) .\end{cases}
$$

(2) $T$ decay:

$$
\begin{gathered}
\Gamma_{T \rightarrow W b}=\frac{g^{2} \delta_{t}^{2} m_{T}^{3}}{64 \pi m_{W}^{2}}\left(1+\frac{m_{W}^{2}}{m_{T}^{2}}-\frac{2 m_{W}^{4}}{m_{T}^{4}}\right) F\left(0, \frac{m_{W}}{m_{T}}\right) \simeq \frac{\delta_{t}^{2} m_{T}^{3}}{16 \pi v^{2}}, \\
\Gamma_{T \rightarrow Z t}=\frac{g^{2} \delta_{t}^{2} m_{T}^{3}}{128 \pi c_{W}^{2} m_{Z}^{2}}\left(1+\frac{m_{Z}^{2}-2 m_{t}^{2}}{m_{T}^{2}}+\frac{m_{t}^{4}+m_{t}^{2} m_{Z}^{2}-2 m_{Z}^{4}}{m_{T}^{4}}\right) F\left(\frac{m_{t}}{m_{T}}, \frac{m_{Z}}{m_{T}}\right) \simeq \frac{\delta_{t}^{2} m_{T}^{3}}{32 \pi v^{2}}, \\
\Gamma_{T \rightarrow H t}=\frac{m_{T}^{3} \delta_{t}^{2}}{32 \pi v^{2}}\left(1+\frac{m_{t}^{2}-m_{H}^{2}}{m_{T}^{2}}\right) F\left(\frac{m_{t}}{m_{T}}, \frac{m_{H}}{m_{T}}\right) \simeq \frac{\delta_{t}^{2} m_{T}^{3}}{32 \pi v^{2}},
\end{gathered}
$$




$$
\Gamma_{T \rightarrow \eta t}=\frac{m_{T} m_{t}^{2}}{32 \pi v^{2}}\left(1+\frac{m_{t}^{2}-m_{\eta}^{2}}{m_{T}^{2}}\right) F\left(\frac{m_{t}}{m_{T}}, \frac{m_{\eta}}{m_{T}}\right) \simeq \frac{m_{T} m_{t}^{2}}{32 \pi v^{2}}\left(1-\frac{m_{\eta}^{2}}{m_{T}^{2}}\right)^{2}
$$

(3) $D, S, N$ decays:

$$
\begin{gathered}
\Gamma_{D \rightarrow W u}=\frac{g^{2} \delta_{D d}^{2} m_{D}^{3}}{64 \pi m_{W}^{2}}\left(1+\frac{m_{W}^{2}}{m_{D}^{2}}-\frac{2 m_{W}^{4}}{m_{D}^{4}}\right) F\left(0, \frac{m_{W}}{m_{D}}\right) \simeq \frac{\delta_{D d}^{2} m_{D}^{3}}{16 \pi v^{2}}, \\
\Gamma_{D \rightarrow Z d}=\frac{g^{2} \delta_{D d}^{2} m_{T}^{3}}{128 \pi c_{W}^{2} m_{Z}^{2}}\left(1+\frac{m_{Z}^{2}}{m_{D}^{2}}-\frac{2 m_{Z}^{4}}{m_{D}^{4}}\right) F\left(0, \frac{m_{Z}}{m_{D}}\right) \simeq \frac{\delta_{D d}^{2} m_{D}^{3}}{32 \pi v^{2}}, \\
\Gamma_{D \rightarrow H d}=\frac{m_{D}^{3} \delta_{D d}^{2}}{32 \pi v^{2}}\left(1-\frac{m_{H}^{2}}{m_{D}^{2}}\right) F\left(0, \frac{m_{H}}{m_{D}}\right) \simeq \frac{\delta_{D d}^{2} m_{D}^{3}}{32 \pi v^{2}}, \\
\Gamma_{D \rightarrow \eta d}=\frac{m_{D}^{3}}{32 \pi v^{2}}\left(\frac{v^{2}}{2 f^{2}}+\delta_{D d}^{2}\right)^{2}\left(1-\frac{m_{\eta}^{2}}{m_{D}^{2}}\right)^{2} \ll \Gamma_{D \rightarrow W u, Z d, H d} .
\end{gathered}
$$

The same formulas hold for $S$ decay channels with the replacements $\delta_{D d} \rightarrow \delta_{S s}, m_{D} \rightarrow m_{S}, D \rightarrow S, d \rightarrow s, u \rightarrow c$. They also hold for $N$ decay channels with the replacements $m_{D} \rightarrow m_{N}, D \rightarrow N, d \rightarrow \nu, u \rightarrow \ell$ and $\delta_{D d} \rightarrow \delta_{D d}^{-}=\frac{v}{\sqrt{2} f t_{\beta}}$.

(4) $Z^{\prime}$ decay:

For $Z^{\prime} \rightarrow f \bar{f}$ decay modes, assuming the interaction Lagrangian $\mathcal{L} \supset \sum_{f} g\left(a_{L}^{f} \bar{f}_{L} \gamma^{\mu} f_{L}+a_{R}^{f} \bar{f}_{R} \gamma^{\mu} f_{R}\right) Z_{\mu}^{\prime}$, the decay width is given by

$$
\Gamma_{Z^{\prime} \rightarrow f \bar{f}}=\frac{N_{c} g^{2} m_{Z^{\prime}}}{24 \pi}\left(\left(\left(a_{L}^{f}\right)^{2}+\left(a_{R}^{f}\right)^{2}\right)\left(1-\frac{m_{f}^{2}}{m_{Z^{\prime}}^{2}}\right)+6 a_{L}^{f} a_{R}^{f} \frac{m_{f}^{2}}{m_{Z^{\prime}}^{2}}\right) \sqrt{1-\frac{4 m_{f}^{2}}{m_{Z^{\prime}}^{2}}}
$$

$N_{c}=1$ for leptons and $N_{c}=3$ for quarks. For SM quarks, we can take $m_{f}=0$ since $m_{Z^{\prime}} \sim \mathcal{O}(f)$. $a_{L}^{f}$ and $a_{R}^{f}$ can be extracted from Eqs. (64)-(66). Thus, the $Z^{\prime} \rightarrow f \bar{f}$ decay widths are

$$
\begin{gathered}
\Gamma_{Z^{\prime} \rightarrow \ell^{+} \ell^{-}}=\frac{g^{2} m_{Z^{\prime}}\left(\left(1-t_{W}^{2}\right)^{2}+4 t_{W}^{4}\right)}{96 \pi\left(3-t_{W}^{2}\right)}, \\
\Gamma_{Z^{\prime} \rightarrow \nu \bar{\nu}}=\frac{g^{2} m_{Z^{\prime}}\left(1-t_{W}^{2}\right)^{2}}{96 \pi\left(3-t_{W}^{2}\right)}, \\
\Gamma_{Z^{\prime} \rightarrow u \bar{u}}=\Gamma_{Z^{\prime} \rightarrow c \bar{c}}=\frac{g^{2} m_{Z^{\prime}}}{72 \pi}\left(\frac{3-t_{W}^{2}}{4}+\frac{4 t_{W}^{4}}{3-t_{W}^{2}}\right), \\
\Gamma_{Z^{\prime} \rightarrow d \bar{d}}=\Gamma_{Z^{\prime} \rightarrow s \bar{s}}=\frac{g^{2} m_{Z^{\prime}}}{72 \pi}\left(\frac{3-t_{W}^{2}}{4}+\frac{t_{W}^{4}}{3-t_{W}^{2}}\right), \\
\Gamma_{Z^{\prime} \rightarrow b \bar{b}}=\frac{g^{2} m_{Z^{\prime}}}{72 \pi\left(3-t_{W}^{2}\right)}\left(\frac{\left(3+t_{W}^{2}\right)^{2}}{4}+t_{W}^{4}\right), \\
\Gamma_{Z^{\prime} \rightarrow t \bar{t}}=\frac{g^{2} m_{Z^{\prime}}}{72 \pi\left(3-t_{W}^{2}\right)}\left(\frac{\left(3+t_{W}^{2}\right)^{2}}{4}+4 t_{W}^{4}\right), \\
\Gamma_{Z^{\prime} \rightarrow N \bar{N}}=\frac{g^{2} m_{Z^{\prime}}}{24 \pi\left(3-t_{W}^{2}\right)}\left(1-\frac{m_{N}^{2}}{m_{Z^{\prime}}^{2}}\right) \sqrt{1-\frac{4 m_{N}^{2}}{m_{Z^{\prime}}^{2}}},
\end{gathered}
$$




$$
\begin{gathered}
\Gamma_{Z^{\prime} \rightarrow D \bar{D}, S \bar{S}}=\frac{g^{2} m_{Z^{\prime}}}{72 \pi\left(3-t_{W}^{2}\right)}\left[\left(\left(3-t_{W}^{2}\right)^{2}+t_{W}^{4}\right)\left(1-\frac{m_{D, S}^{2}}{m_{Z^{\prime}}^{2}}\right)-6 t_{W}^{2}\left(3-t_{W}^{2}\right) \frac{m_{D, S}^{2}}{m_{Z^{\prime}}^{2}}\right] \sqrt{1-\frac{4 m_{D, S}^{2}}{m_{Z^{\prime}}^{2}}}, \\
\Gamma_{Z^{\prime} \rightarrow T \bar{T}}=\frac{g^{2} m_{Z^{\prime}}}{72 \pi\left(3-t_{W}^{2}\right)}\left[\left(\left(3-2 t_{W}^{2}\right)^{2}+4 t_{W}^{4}\right)\left(1-\frac{m_{T}^{2}}{m_{Z^{\prime}}^{2}}\right)-12 t_{W}^{2}\left(3-2 t_{W}^{2}\right) \frac{m_{T}^{2}}{m_{Z^{\prime}}^{2}}\right] \sqrt{1-\frac{4 m_{T}^{2}}{m_{Z^{\prime}}^{2}}} .
\end{gathered}
$$

Decay widths in bosonic channels:

$$
\begin{gathered}
\Gamma_{Z^{\prime} \rightarrow W^{+} W^{-}}=\frac{g^{2} m_{Z^{\prime}}\left(1-t_{W}^{2}\right)^{2}}{192 \pi\left(3-t_{W}^{2}\right)}\left(1-\frac{4 m_{W}^{2}}{m_{Z^{\prime}}^{2}}\right)^{\frac{3}{2}}\left(1+\frac{20 m_{W}^{2}}{m_{Z^{\prime}}^{2}}+\frac{12 m_{W}^{4}}{m_{Z^{\prime}}^{4}}\right) \simeq \frac{g^{2} m_{Z^{\prime}}\left(1-t_{W}^{2}\right)^{2}}{192 \pi\left(3-t_{W}^{2}\right)}, \\
\Gamma_{Z^{\prime} \rightarrow Z H}=\frac{g^{2} m_{Z^{\prime}}\left(1-t_{W}^{2}\right)^{2}}{192 \pi\left(3-t_{W}^{2}\right)} F\left(\frac{m_{Z}}{m_{Z^{\prime}}}, \frac{m_{H}}{m_{Z^{\prime}}}\right)\left[F^{2}\left(\frac{m_{Z}}{m_{Z^{\prime}}}, \frac{m_{H}}{m_{Z^{\prime}}}\right)+\frac{12 m_{Z}^{2}}{m_{Z^{\prime}}^{2}}\right] \simeq \frac{g^{2} m_{Z^{\prime}}\left(1-t_{W}^{2}\right)^{2}}{192 \pi\left(3-t_{W}^{2}\right)}, \\
\Gamma_{Z^{\prime} \rightarrow H \eta}=\frac{g^{2} m_{Z^{\prime}}(v / f)^{2}}{24 \pi\left(3-t_{W}^{2}\right) t_{2 \beta}^{2}} F^{3}\left(\frac{m_{H}}{m_{Z^{\prime}}}, \frac{m_{\eta}}{m_{Z^{\prime}}}\right), \\
\Gamma_{Z^{\prime} \rightarrow Y \eta}=\frac{g^{2} m_{Z^{\prime}}(v / f)^{2}}{384 \pi c_{W}^{4}} F\left(\frac{m_{Y}}{m_{Z^{\prime}}}, \frac{m_{\eta}}{m_{Z^{\prime}}}\right)\left(2+\frac{\left(m_{Z^{\prime}}^{2}+m_{Y}^{2}-m_{\eta}^{2}\right)^{2}}{4 m_{Z^{\prime}}^{2} m_{Y}^{2}}\right) .
\end{gathered}
$$

[1] G. Aad et al. (ATLAS Collaboration), Phys. Lett. B 716, 1 (2012).

[2] S. Chatrchyan et al. (CMS Collaboration), Phys. Lett. B 716, 30 (2012).

[3] A. N. Schellekens, Rev. Mod. Phys. 85, 1491 (2013).

[4] J. F. Donoghue, Annu. Rev. Nucl. Part. Sci. 66, 1 (2016).

[5] N. Arkani-Hamed, A. G. Cohen, and H. Georgi, Phys. Lett. B 513, 232 (2001).

[6] N. Arkani-Hamed, A. G. Cohen, T. Gregoire, and J. G. Wacker, J. High Energy Phys. 08 (2002) 020.

[7] N. Arkani-Hamed, A. G. Cohen, E. Katz, A. E. Nelson, T. Gregoire, and J. G. Wacker, J. High Energy Phys. 08 (2002) 021.

[8] N. Arkani-Hamed, A. G. Cohen, E. Katz, and A. E. Nelson, J. High Energy Phys. 07 (2002) 034.

[9] M. Schmaltz and D. Tucker-Smith, Annu. Rev. Nucl. Part. Sci. 55, 229 (2005).

[10] M. Perelstein, Prog. Part. Nucl. Phys. 58, 247 (2007).

[11] D. Dercks, G. Moortgat-Pick, J. Reuter, and S. Y. Shim, J. High Energy Phys. 05 (2018) 049.

[12] J. Reuter and M. Tonini, J. High Energy Phys. 02 (2013) 077.

[13] J. Reuter, M. Tonini, and M. de Vries, J. High Energy Phys. 02 (2014) 053.

[14] X.-F. Han, L. Wang, J. M. Yang, and J. Zhu, Phys. Rev. D 87, 055004 (2013).

[15] D. E. Kaplan and M. Schmaltz, J. High Energy Phys. 10 (2003) 039.

[16] M. Schmaltz, J. High Energy Phys. 08 (2004) 056.
[17] W. Kilian, D. Rainwater, and J. Reuter, Phys. Rev. D 71, 015008 (2005).

[18] W. Kilian, D. Rainwater, and J. Reuter, Phys. Rev. D 74, 095003 (2006); 74, 099905(E) (2006).

[19] K. Cheung, S.-P. He, Y.-n. Mao, C. Zhang, and Y. Zhou, Phys. Rev. D 97, 115001 (2018).

[20] K. Cheung and J. Song, Phys. Rev. D 76, 035007 (2007).

[21] K. Cheung, J. Song, P. Tseng, and Q.-S. Yan, Phys. Rev. D 78, 055015 (2008).

[22] S.-P. He, Y.-n. Mao, C. Zhang, and S.-h. Zhu, Phys. Rev. D 97, 075005 (2018).

[23] F. del Aguila, J. I. Illana, and M. D. Jenkins, J. High Energy Phys. 03 (2011) 080.

[24] T. Han, H. E. Logan, and L.-T. Wang, J. High Energy Phys. 01 (2006) 099.

[25] O. C. W. Kong, J. Korean Phys. Soc. 45, S404 (2004).

[26] O. C. W. Kong, Phys. Rev. D 70, 075021 (2004).

[27] Y.-n. Mao, Phys. Rev. D 97, 075031 (2018).

[28] F. del Aguila, M. Masip, and J. L. Padilla, Phys. Lett. B 627, 131 (2005).

[29] M. Aaboud et al. (ATLAS Collaboration), J. High Energy Phys. 10 (2017) 182.

[30] A. M. Sirunyan et al. (CMS Collaboration), J. High Energy Phys. 06 (2018) 120.

[31] C. S. Kim, K. Y. Lee, and J. Park, Phys. Rev. D 85, 117702 (2012).

[32] S. Weinberg, The Quantum Theory of Fields. Vol. 1: Foundations (Cambridge University Press, Cambridge, England, 2005). 
[33] J. Erler and A. Freitas, http://pdg.lbl.gov/2018/reviews/ rpp2018-rev-standard-model.pdf.

[34] A. M. Sirunyan et al. (CMS Collaboration), Phys. Rev. D 97, 072008 (2018).

[35] N. Bizot, G. Cacciapaglia, and T. Flacke, J. High Energy Phys. 06 (2018) 065.

[36] J. Serra, J. High Energy Phys. 09 (2015) 176.

[37] J. Kearney, A. Pierce, and J. Thaler, J. High Energy Phys. 10 (2013) 230.
[38] J. Alwall, R. Frederix, S. Frixione, V. Hirschi, F. Maltoni, O. Mattelaer, H.-S. Shao, T. Stelzer, P. Torrielli, and M. Zaro, J. High Energy Phys. 07 (2014) 079.

[39] A. Alloul, N. D. Christensen, C. Degrande, C. Duhr, and B. Fuks, Comput. Phys. Commun. 185, 2250 (2014).

[40] A. D. Martin, W. J. Stirling, R. S. Thorne, and G. Watt, Eur. Phys. J. C 63, 189 (2009).

[41] D. E. Kaplan, K. Rehermann, M. D. Schwartz, and B. Tweedie, Phys. Rev. Lett. 101, 142001 (2008). 